\title{
FINITE ELEMENT APPROXIMATION OF A STEFAN PROBLEM WITH DEGENERATE JOULE HEATING*
}

\author{
JOHN W. BARRETT ${ }^{1}$ AND ROBERT NÜRNBERG ${ }^{1}$
}

\begin{abstract}
We consider a fully practical finite element approximation of the following degenerate system

$$
\frac{\partial}{\partial t} \rho(u)-\nabla \cdot(\alpha(u) \nabla u) \ni \sigma(u)|\nabla \phi|^{2}, \quad \nabla \cdot(\sigma(u) \nabla \phi)=0
$$

subject to an initial condition on the temperature, $u$, and boundary conditions on both $u$ and the electric potential, $\phi$. In the above $\rho(u)$ is the enthalpy incorporating the latent heat of melting, $\alpha(u)>0$ is the temperature dependent heat conductivity, and $\sigma(u) \geq 0$ is the electrical conductivity. The latter is zero in the frozen zone, $u \leq 0$, which gives rise to the degeneracy in this Stefan system. In addition to showing stability bounds, we prove (subsequence) convergence of our finite element approximation in two and three space dimensions. The latter is non-trivial due to the degeneracy in $\sigma(u)$ and the quadratic nature of the Joule heating term forcing the Stefan problem. Finally, some numerical experiments are presented in two space dimensions.
\end{abstract}

Mathematics Subject Classification. 35K55, 35K65, 35R35, 65M12, 65M60, 80A22.

Received: July 29, 2003. Revised: April 7, 2004.

\section{INTRODUCTION}

In situ vitrification (ISV) is a thermal treatment process that converts contaminated soil back into a durable leach resistant product. In [7], this process is described as follows. Electrodes are inserted into the soil to the desired treatment depth and a layer of electrically conductive material (a "starter path") is placed between the electrodes. Electric power supplied to the electrodes causes the conductive material to melt, thus melting the surrounding soil. Electrical energy is transferred to the molten soil through Joule (resistance) heating, and the soil continues to melt to the desired depth, at which time the power to the electrodes is discontinued. After completion of the melt, the molten soil cools and solidifies. The product resulting from this ISV process is a glass and crystalline mass, resembling natural obsidian. Hence the contaminated materials in the original soil are now trapped in this resulting solid, which is leach resistant.

A simplified mathematical model of the steady state problem is considered in [6]. In this paper, we consider the corresponding time dependent model studied in [10].

Keywords and phrases. Stefan problem, Joule heating, degenerate system, finite elements, convergence.

* Part of this work was carried out while the authors participated in the 2003 programme Computational Challenges in Partial Differential Equations at the Isaac Newton Institute, Cambridge, UK.

1 Department of Mathematics, Imperial College, London, SW7 2AZ, UK. e-mail: jwb@ic.ac.uk 
Let $\Omega \subset \mathbb{R}^{d}, d=2$ or 3 , be the spatial domain of interest, the region of soil, with boundary $\partial \Omega$. For simplicity in describing the finite element partitioning, we make the following assumption on $\Omega$ throughout:

(A1) $\Omega$ is polygonal, if $d=2$; and polyhedral, if $d=3$.

However, for ease of exposition in this paper, we consider the specific situation in the figure below, i.e. $\Omega$ takes the form of a rectangle minus two rectangular electrodes if $d=2$; and a cuboid minus two cuboidal electrodes if $d=3$.

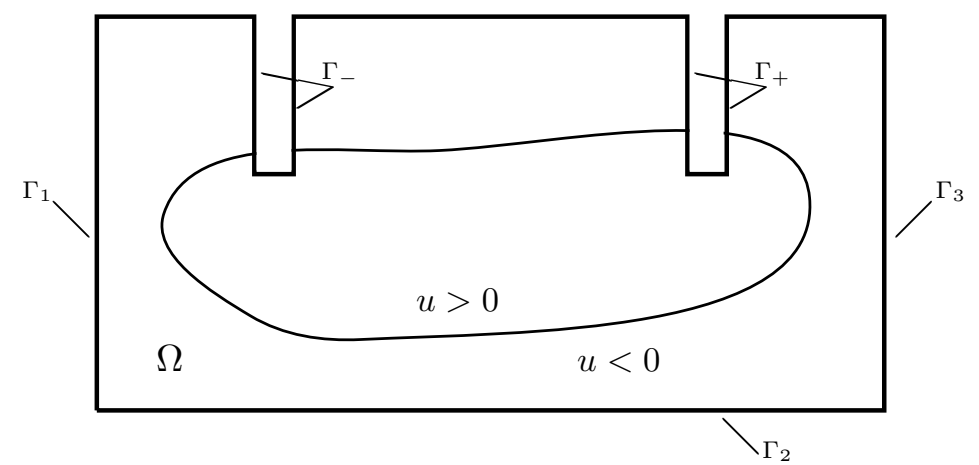

On noting the figure above, where $\Gamma_{ \pm}$represents the boundary of the \pm ive electrode, we define

$$
\Gamma_{D}^{\phi}:=\bar{\Gamma}_{+} \cup \bar{\Gamma}_{-}, \quad \Gamma_{N}^{\phi}:=\partial \Omega \backslash \Gamma_{D}^{\phi}, \quad \Gamma_{D}^{u}:=\bar{\Gamma}_{1} \cup \bar{\Gamma}_{2} \cup \bar{\Gamma}_{3}, \quad \Gamma_{N}^{u}:=\partial \Omega \backslash \Gamma_{D}^{u}
$$

These correspond to parts of the boundary, where we will prescribe Dirichlet and Neumann conditions, respectively, for the electric potential $\phi$ and the temperature $u$.

Then [10] proposes the following nonlinear degenerate parabolic system as a simplified model of the ISV process:

(P) Find functions $u, v, \phi: \Omega \times[0, T] \rightarrow \mathbb{R}$ such that $v \in \rho(u)$ for a.e. $(x, t) \in \Omega_{T}$ and

$$
\begin{array}{rlrl}
\frac{\partial v}{\partial t}-\nabla \cdot(\alpha(u) \nabla u) & =\sigma(u)|\nabla \phi|^{2} & & \text { in } \Omega_{T}, \\
u=\bar{u}_{D} \quad \text { on } \Gamma_{D}^{u} \times(0, T], \quad \alpha(u) \frac{\partial u}{\partial \nu}=-\gamma\left(u-\bar{u}_{N}\right) & & \text { on } \Gamma_{N}^{u} \times(0, T], \\
v(\cdot, 0) & =v^{0}(\cdot) & & \text { in } \Omega, \\
\nabla \cdot(\sigma(u) \nabla \phi) & =0 & & \text { in } \Omega_{T}, \\
\phi=\bar{\phi} \quad \text { on } \Gamma_{D}^{\phi} \times(0, T], \quad \sigma(u) \frac{\partial \phi}{\partial \nu}=0 & & \text { on } \Gamma_{N}^{\phi} \times(0, T] ;
\end{array}
$$

where $T>0$ is a fixed positive time, $\Omega_{T}:=\Omega \times(0, T]$ and $\nu$ is the outward unit normal to $\partial \Omega$. In $(1.1 \mathrm{a}-\mathrm{e}) \bar{u}_{D}$ and $\bar{u}_{N}, \gamma$ represent the $\Gamma_{D}^{u}$ trace and $\Gamma_{N}^{u}$ traces, respectively, of given functions

$$
\bar{u}_{D}, \bar{u}_{N}, \gamma \in W^{1, r}(\Omega) \subset C(\bar{\Omega}), \quad r>d ; \quad \text { with }\left.\quad \gamma\right|_{\Gamma_{N}^{u}} \geq 0
$$

and $\bar{\phi}$ represents the $\Gamma_{D}^{\phi}$ trace of a given function $\bar{\phi} \in C\left([0, T] ; W^{1, \infty}\left(\Omega_{T}\right)\right)$ satisfying

$$
m_{\phi} \leq \bar{\phi}_{m}(t) \leq \bar{\phi}(x, t) \leq \bar{\phi}_{M}(t) \leq M_{\phi} \quad \text { for a.e. }(x, t) \in \Omega_{T},
$$

where $m_{\phi}, M_{\phi} \in \mathbb{R}$. Here $\bar{\phi}$ is allowed to be time dependent in order to model the turning on and off of the power supply to the electrodes. Note that $\bar{u}_{D}$ and $\bar{u}_{N}$, on the other hand, are time independent, because we 
assume that (i) the subsurface boundary $\Gamma_{D}^{u}$ is sufficiently far away from the electrodes to be effected by the electric current and remains solid throughout; and (ii) the electrodes and the top surface (air) have known time independent temperature $\bar{u}_{N}$ but heat exchange into the medium is allowed via (1.1b).

Furthermore, the enthalpy or heat content, $v$, is defined in terms of the temperature and the given parameters: latent heat, $\lambda \in \mathbb{R}_{\geq 0}:=\{s \in \mathbb{R}: s \geq 0\}$, and heat capacities, $\rho_{ \pm} \in \mathbb{R}_{>0}:=\{s \in \mathbb{R}: s>0\}$; by

$$
\rho(s):= \begin{cases}\rho_{+} s+\lambda & \text { if } s>0 \\ {[0, \lambda]} & \text { if } s=0 \\ \rho_{-} s & \text { if } s<0\end{cases}
$$

Here we have assumed, without loss of generality on rescaling, that zero is the phase change temperature. For later use, we introduce the monotone function $\psi: \mathbb{R} \rightarrow \mathbb{R}$, and its antiderivative $\Psi$, defined for all $s \in \mathbb{R}$ by

$$
\psi(s):=\rho^{-1}(s) \quad \text { and } \quad \Psi(s):=\int_{0}^{s} \psi(q) \mathrm{d} q \quad \Longrightarrow \quad C_{1} s^{2}-C_{2} \leq \Psi(s) \leq C_{3} s^{2} ;
$$

where $C_{i}\left(\lambda, \rho_{ \pm}\right) \in \mathbb{R}_{>0}$. Finally, $\alpha \in C^{0,1}(\mathbb{R})$ is the given temperature dependent heat conductivity with

$$
0<m_{\alpha} \leq \alpha(s) \leq M_{\alpha} \quad \forall s \in \mathbb{R}
$$

and $\sigma \in C^{0,1}(\mathbb{R})$ is the given temperature dependent electrical conductivity satisfying

$$
\sigma(s)=0, \quad \text { for } s \leq 0 ; \quad 0 \leq \sigma(s) \leq M_{\sigma}, \quad \text { for } s>0 ; \quad \int_{0}^{1}[\sigma(s)]^{-\frac{1}{2}} \mathrm{~d} s=\infty .
$$

As a possible example, let $\sigma(s)$, for $s \geq 0$, be given by

$$
\sigma(s)=\left\{\begin{array}{ll}
\sigma_{0} s_{0}^{p} & \text { if } s \geq s_{0}, \\
\sigma_{0} s^{p} & \text { if } s \in\left[0, s_{0}\right],
\end{array} \quad \text { where } p \geq 2 \text { and } s_{0}, \sigma_{0} \in \mathbb{R}_{>0}\right.
$$

(P) models the combined process of heat conduction and electrical conduction in a body, which may undergo a phase change as a result of heat generated by the current. The rate of energy generation associated with electrical current flow, the so called Joule heating, is represented by the term $\sigma(u)|\nabla \phi|^{2}$ on the right hand side of (1.1a).

The fact that $\sigma(u)$ vanishes in the frozen zone, $\{u \leq 0\}$, gives rise to the degeneracy in this Stefan system. Existence of a solution to $(\mathrm{P})$ is non-trivial due to this degeneracy of $\sigma(u)$ and the quadratic nature of the Joule term forcing the Stefan problem. Existence of a weak solution to the steady state version of $(\mathrm{P})$ can be found in [6], and to $(\mathrm{P})$ in [10]. It is the goal of this paper to adapt the techniques in [10], in order to prove (subsequence) convergence of a fully practical finite element approximation of $(\mathrm{P})$. In particular, the integral condition on $\sigma$ in (1.6) plays a key role; see Lemma 3.4 below. Furthermore, for the analysis in [10] it is crucial to rewrite the right hand side term of the weak formulation of (1.1a), on noting (1.1d), as

$$
\begin{aligned}
\int_{\Omega_{T}} \sigma(u)|\nabla \phi|^{2} \eta \mathrm{d} x \mathrm{~d} t & =\int_{\Omega_{T}} \sigma(u) \nabla \phi[\eta \nabla \bar{\phi}+\nabla(\eta(\phi-\bar{\phi}))-(\phi-\bar{\phi}) \nabla \eta] \mathrm{d} x \mathrm{~d} t \\
& =\int_{\Omega_{T}} \sigma(u) \nabla \phi[\eta \nabla \bar{\phi}-(\phi-\bar{\phi}) \nabla \eta] \mathrm{d} x \mathrm{~d} t \quad \forall \eta \in L^{2}\left(0, T ; H^{1}(\Omega)\right) .
\end{aligned}
$$

Given that a priori one can only show that $\sigma(u)|\nabla \phi|^{2} \in L^{\infty}\left(0, T ; L^{1}(\Omega)\right.$ ), one would need an (unavailable) $L^{\infty}\left(\Omega_{T}\right)$ bound on $u$, in order to establish the desired $L^{2}\left(0, T ; H^{1}(\Omega)\right)$ bound on $u$ directly from using the first integral in (1.8) with $\eta=u-\bar{u}_{D}$. However an $L^{\infty}\left(\Omega_{T}\right)$ bound is available, via a weak maximum principle, 
on $\phi$. Hence the identity (1.8) does now yield the desired $L^{2}\left(0, T ; H^{1}(\Omega)\right)$ bound on $u$. To transfer the described strategy to the discrete level is rather delicate. In order to do so we have to modify the natural finite element approximation of (1.1a) and introduce matrices $D(\cdot)$; see (2.14) below. Finally, we note that our convergence analysis is restricted to subsequences due to the lack of a uniqueness proof for the weak solution to $(\mathrm{P})$.

Although there is considerable numerical analysis on the non-degenerate system; see e.g. [5], where error bounds for a fully discrete finite element approximation of $(\mathrm{P})$ with $\lambda=0, \rho_{ \pm}=1, \alpha(\cdot) \equiv 1$ and $\sigma(\cdot) \geq c_{m}>0$ are derived; we know of no numerical analysis on the degenerate system $(\mathrm{P})$. In addition, we believe that the techniques used here on this model problem will be applicable to similar degenerate systems. Finally, we note that a related Stefan system modelling the artificial freezing of water-saturated soil is studied numerically in [1]. There the Joule heating effect in (1.1a) is replaced by a convection term $v \cdot \nabla u$, where the velocity field $v$ of the groundwater is coupled to the pressure $\phi$ through Darcy's law, $v=\sigma(u) \nabla \phi$, and $\phi$ satisfies (1.1b) with the permeability $\sigma(\cdot)$ vanishing in the frozen region.

This paper is organized as follows. In Section 2 we formulate a fully practical finite element approximation of the degenerate system (P). In Section 3 we prove (subsequence) convergence in two and three space dimensions. Finally, in Section 4 we present some numerical experiments in two space dimensions.

\section{Notation and auxiliary results}

Let $D \subset \mathbb{R}^{d}, d=1,2$ or 3 , with a Lipschitz boundary $\partial D$ if $d=2$ or 3 . We adopt the standard notation for Sobolev spaces, denoting the norm of $W^{m, q}(D)(m \in \mathbb{N}, q \in[1, \infty])$ by $\|\cdot\|_{m, q, D}$ and the semi-norm by $|\cdot|_{m, q, D}$. We extend these norms and semi-norms in the natural way to the corresponding spaces of vector and matrix valued functions. For $q=2, W^{m, 2}(D)$ will be denoted by $H^{m}(D)$ with the associated norm and semi-norm written as, respectively, $\|\cdot\|_{m, D}$ and $|\cdot|_{m, D}$. For notational convenience, we drop the domain subscript on the above norms and semi-norms in the case $D \equiv \Omega$. Throughout $(\cdot, \cdot)$ denotes the standard $L^{2}$ inner product over $\Omega$. In addition we define

$$
f \eta:=\frac{1}{\underline{m}(\Omega)}(\eta, 1) \quad \forall \eta \in L^{1}(\Omega) \quad \text { and } \quad f_{\kappa} \eta:=\frac{1}{\underline{m}(\kappa)} \int_{\kappa} \eta \mathrm{d} x \quad \forall \eta \in L^{1}(\kappa),
$$

where $\underline{m}(D)$ denotes the measure of $D$.

We recall the following compactness result. Let $X, Y$ and $Z$ be Banach spaces with a compact embedding $X \hookrightarrow Y$ and a continuous embedding $Y \hookrightarrow Z$. Then any bounded and closed subset $E$ of $L^{2}(0, T ; X)$ with

$$
\lim _{\theta \rightarrow 0}\left\{\sup _{\eta \in E}\|\eta(\cdot, \cdot+\theta)-\eta(\cdot, \cdot)\|_{L^{2}(0, T-\theta ; Z)}\right\}=0
$$

is compact in $L^{2}(0, T ; Y)$, see [8]. In addition, we note the following Egoroff type result (see [10], Theorem C). If $\left\{z_{k}\right\}_{k \geq 0}$ is bounded in $L^{q}\left(0, T ; W^{1, q}(\Omega)\right)$ and precompact in $L^{q}\left(\Omega_{T}\right)$, then for each $s \in[1, q)$ there exists a subsequence $\left\{z_{k_{j}}\right\}_{j \geq 0}$ and a function $z \in L^{q}\left(0, T ; W^{1, q}(\Omega)\right)$ such that for all $\varepsilon>0$ there corresponds a function $\vartheta_{\varepsilon} \in L^{s}\left(0, T ; W^{1, s}(\Omega)\right)$ such that

$$
\begin{aligned}
& z_{k_{j}} \rightarrow z \quad \text { uniformly on }\left\{\vartheta_{\varepsilon}>0\right\} \quad \text { as } j \rightarrow \infty \\
& 0 \leq \vartheta_{\varepsilon}(x, t) \leq 1 \quad \text { for a.e. }(x, t) \in \Omega_{T} \quad \text { and } \quad\left\|1-\vartheta_{\varepsilon}\right\|_{L^{s}\left(\Omega_{T}\right)}^{s}+\left\|\nabla \vartheta_{\varepsilon}\right\|_{L^{s}\left(\Omega_{T}\right)}^{s} \leq \varepsilon .
\end{aligned}
$$

Throughout $C$ denotes a generic constant independent of $h, \tau$ and $\delta$; the mesh and temporal discretization parameters and the regularization parameter. In addition $C\left(a_{1}, \cdots, a_{I}\right)$ denotes a constant depending on the arguments $\left\{a_{i}\right\}_{i=1}^{I}$. Furthermore $\cdot{ }^{(\star)}$ denotes an expression with or without the superscript $\star$. Finally, we define for any $s \in \mathbb{R}$

$$
[s]_{+}:=\max \{s, 0\} .
$$




\section{Finite element approximation}

We consider the finite element approximation of $(\mathrm{P})$ under the following assumptions on the mesh:

(A2) Let $\Omega$ be given as in (A1). Let $\left\{\mathcal{T}^{h}\right\}_{h>0}$ be a regular family of partitionings of $\Omega$ into disjoint open simplices $\kappa$ with $h_{\kappa}:=\operatorname{diam}(\kappa)$ and $h:=\max _{\kappa \in \mathcal{T}^{h}} h_{\kappa}$, so that $\bar{\Omega}=\cup_{\kappa \in \mathcal{T}^{h}} \bar{\kappa}$. In addition, it is assumed that $\mathcal{T}^{h}$ is a (weakly) acute partitioning; that is for (a) $d=2$, for any pair of adjacent triangles the sum of opposite angles relative to the common side does not exceed $\pi$; (b) $d=3$, the angle between any faces of the same tetrahedron does not exceed $\frac{\pi}{2}$.

Associated with $\mathcal{T}^{h}$ is the finite element space

$$
S^{h}:=\left\{\chi \in C(\bar{\Omega}):\left.\chi\right|_{\kappa} \text { is linear } \forall \kappa \in \mathcal{T}^{h}\right\} \subset H^{1}(\Omega) .
$$

Let $J$ be the set of nodes of $\mathcal{T}^{h}$ and $\left\{p_{j}\right\}_{j \in J}$ the coordinates of these nodes. Let $\left\{\chi_{j}\right\}_{j \in J}$ be the standard basis functions for $S^{h}$; that is $\chi_{j} \in S^{h}$ and $\chi_{j}\left(p_{i}\right)=\delta_{i j}$ for all $i, j \in J$. We introduce $\pi^{h}: C(\bar{\Omega}) \rightarrow S^{h}$, the interpolation operator, such that $\left(\pi^{h} \eta\right)\left(p_{j}\right)=\eta\left(p_{j}\right)$ for all $j \in J$. A discrete semi-inner product on $C(\bar{\Omega})$ is then defined by

$$
\left(\eta_{1}, \eta_{2}\right)^{h}:=\int_{\Omega} \pi^{h}\left[\eta_{1}(x) \eta_{2}(x)\right] \mathrm{d} x=\sum_{j \in J} m_{j} \eta_{1}\left(p_{j}\right) \eta_{2}\left(p_{j}\right), \quad \text { where } \quad m_{j}:=\left(1, \chi_{j}\right)>0 .
$$

The induced discrete semi-norm is then $|\eta|_{h}:=\left[(\eta, \eta)^{h}\right]^{\frac{1}{2}}$, where $\eta \in C(\bar{\Omega})$.

We note that the (weak) acuteness assumption yields that

$$
\int_{\kappa} \nabla \chi_{i} \cdot \nabla \chi_{j} \mathrm{~d} x \leq 0 \quad i \neq j, \quad \forall \kappa \in \mathcal{T}^{h}
$$

Let $f \in C^{0,1}(\mathbb{R})$ be monotone with Lipschitz constant $L_{f}$, then it follows from $(2.3)$ and the inequality

$$
(f(a)-f(b))^{2} \leq L_{f}(f(a)-f(b))(a-b) \quad \forall a, b \in \mathbb{R}
$$

that for all $\chi \in S^{h}$

$$
\int_{\kappa}\left|\nabla \pi^{h}[f(\chi)]\right|^{2} \mathrm{~d} x \leq L_{f} \int_{\kappa} \nabla \chi \cdot \nabla \pi^{h}[f(\chi)] \mathrm{d} x \quad \forall \kappa \in \mathcal{T}^{h} .
$$

Furthermore, it is easily established (see e.g. [4], p. 69) that for all $\kappa \in \mathcal{T}^{h}$ and for all $\chi \in S^{h}$

$$
\left|\left(I-\pi^{h}\right)[f(\chi)]\right|_{0, \infty, \kappa} \leq h_{\kappa}\left|\nabla\left(\pi^{h}[f(\chi)]\right)\right|_{0, \infty, \kappa} \quad \text { and } \quad\left|f(\chi)-f_{\kappa} \pi^{h}[f(\chi)]\right|_{0, \infty, \kappa} \leq h_{\kappa}\left|\nabla\left(\pi^{h}[f(\chi)]\right)\right|_{0, \infty, \kappa} .
$$

Next we introduce

$$
\begin{aligned}
H_{\phi(t)}^{1}(\Omega):=\left\{\eta \in H^{1}(\Omega): \eta(\cdot)=\bar{\phi}(\cdot, t) \text { on } \Gamma_{D}^{\phi}\right\}, & H_{\phi, 0}^{1}(\Omega):=\left\{\eta \in H^{1}(\Omega): \eta=0 \text { on } \Gamma_{D}^{\phi}\right\} \\
H_{u}^{1}(\Omega):=\left\{\eta \in H^{1}(\Omega): \eta=\bar{u}_{D} \text { on } \Gamma_{D}^{u}\right\}, & H_{u, 0}^{1}(\Omega):=\left\{\eta \in H^{1}(\Omega): \eta=0 \text { on } \Gamma_{D}^{u}\right\}
\end{aligned}
$$

In addition to $\mathcal{T}^{h}$, let $0=t_{0}<t_{1}<\ldots<t_{N-1}<t_{N}=T$ be a partitioning of $[0, T]$ into possibly variable time steps $\tau_{n}:=t_{n}-t_{n-1}, n=1 \rightarrow N$. We set $\tau:=\max _{n=1 \rightarrow N} \tau_{n}$. On noting $(2.6 \mathrm{a}, \mathrm{b})$ and $(2.1)$, we then introduce

$$
\begin{aligned}
S_{\phi}^{h, n}:=\left\{\chi \in S^{h}: \chi=\pi^{h} \bar{\phi}^{n} \text { on } \Gamma_{D}^{\phi}\right\}, & S_{\phi, 0}^{h}:=\left\{\chi \in S^{h}: \chi=0 \text { on } \Gamma_{D}^{\phi}\right\} \subset H_{\phi, 0}^{1}(\Omega) ; \\
S_{u}^{h}:=\left\{\chi \in S^{h}: \chi=\pi^{h} \bar{u}_{D} \text { on } \Gamma_{D}^{u}\right\}, & S_{u, 0}^{h}:=\left\{\chi \in S^{h}: \chi=0 \text { on } \Gamma_{D}^{u}\right\} \subset H_{u, 0}^{1}(\Omega)
\end{aligned}
$$


where $\bar{\phi}^{n}(\cdot):=\bar{\phi}\left(\cdot, t_{n}\right)$. Furthermore, given a regularization parameter $\delta \in \mathbb{R}_{>0}$, we introduce, on recalling (1.6), (1.9) and (1.5), the discrete (regularized) functions $\sigma_{\delta}^{h}, \alpha^{h}: S^{h} \rightarrow L^{\infty}(\Omega)$ such that for all $\kappa \in \mathcal{T}^{h}$ and $\chi \in S^{h}$

$$
\left.\sigma_{\delta}^{h}(\chi)\right|_{\kappa}:=\delta+f_{\kappa} \pi^{h}[\sigma(\chi)] \quad \text { and }\left.\quad \alpha^{h}(\chi)\right|_{\kappa}:=f_{\kappa} \pi^{h}[\alpha(\chi)] .
$$

In order to formulate our finite element approximation we introduce the following matrices $D(\cdot)$. Let $\left\{e_{i}\right\}_{i=1}^{d}$ be the orthonormal vectors in $\mathbb{R}^{d}$, such that the $j$ th component of $e_{i}$ is $\delta_{i j}, i, j=1 \rightarrow d$. Let $\widehat{\kappa}$ be the standard reference simplex in $\mathbb{R}^{d}$ with vertices $\left\{\widehat{p}_{i}\right\}_{i=0}^{d}$, where $\widehat{p}_{0}$ is the origin and $\widehat{p}_{i}=e_{i}, i=1 \rightarrow d$. Given a $\kappa \in \mathcal{T}^{h}$ with vertices $\left\{p_{i}\right\}_{i=0}^{d}$ there exists a matrix $B_{\kappa}$ such that the mapping $\mathcal{F}_{\kappa}: \widehat{x} \in \mathbb{R}^{d} \rightarrow b_{\kappa}+B_{\kappa} \widehat{x} \in \mathbb{R}^{d}$ maps the vertex $\widehat{p}_{i}$ to $p_{i}, i=0 \rightarrow d$, and hence $\widehat{\kappa}$ to $\kappa$. For all $\kappa \in \mathcal{T}^{h}$ and $\eta \in C(\bar{\kappa})$, we set

$$
\widehat{\eta}(\widehat{x}) \equiv \eta\left(\mathcal{F}_{\kappa} \widehat{x}\right) \quad \text { and } \quad\left(\widehat{\pi}^{h} \widehat{\eta}\right)(\widehat{x}) \equiv\left(\pi^{h} \eta\right)\left(\mathcal{F}_{\kappa} \widehat{x}\right) \quad \forall \widehat{x} \in \bar{\kappa} .
$$

We have for any $z^{h} \in S^{h}$ and $\kappa \in \mathcal{T}^{h}$ that

$$
\nabla z^{h} \equiv B_{\kappa}^{-T} \widehat{\nabla} \widehat{z}^{h}
$$

where $x \equiv\left(x_{1}, \cdots, x_{d}\right)^{T}, \nabla \equiv\left(\frac{\partial}{\partial x_{1}}, \cdots, \frac{\partial}{\partial x_{d}}\right)^{T}, \widehat{x} \equiv\left(\widehat{x}_{1}, \cdots, \widehat{x}_{d}\right)^{T}$ and $\hat{\nabla} \equiv\left(\frac{\partial}{\partial \widehat{x}_{1}}, \cdots, \frac{\partial}{\partial \widehat{x}_{d}}\right)^{T}$. From $(2.9)$ and (2.10), it follows for all $\kappa \in \mathcal{T}^{h}, \eta_{j} \in C(\bar{\kappa})$ and $i=1 \rightarrow d$ that

$$
\begin{aligned}
\frac{\partial}{\partial \widehat{x}_{i}}\left(\widehat{\pi}^{h}\left[\widehat{\eta}_{1} \widehat{\eta}_{2}\right]\right) & =\widehat{\eta}_{1}\left(\widehat{p}_{i}\right) \widehat{\eta}_{2}\left(\widehat{p}_{i}\right)-\widehat{\eta}_{1}\left(\widehat{p}_{i-1}\right) \widehat{\eta}_{2}\left(\widehat{p}_{i-1}\right) \\
& =\left(\widehat{\eta}_{1}\left(\widehat{p}_{i-1}\right)+\widehat{\eta}_{1}\left(\widehat{p}_{i}\right)\right)\left[\widehat{\eta}_{2}\left(\widehat{p}_{i}\right)-\widehat{\eta}_{2}\left(\widehat{p}_{i-1}\right)\right]+\left(\widehat{\eta}_{2}\left(\widehat{p}_{i-1}\right)+\widehat{\eta}_{2}\left(\widehat{p}_{i}\right)\right)\left[\widehat{\eta}_{1}\left(\widehat{p}_{i}\right)-\widehat{\eta}_{1}\left(\widehat{p}_{i-1}\right)\right] .
\end{aligned}
$$

Therefore (2.11) yields for all $\kappa \in \mathcal{T}^{h}$ and $\eta_{j} \in C(\bar{\kappa})$ that

$$
\widehat{\nabla}\left(\widehat{\pi}^{h}\left[\widehat{\eta}_{1} \widehat{\eta}_{2}\right]\right)=\widehat{D}\left(\widehat{\pi}^{h} \widehat{\eta}_{1}\right) \widehat{\nabla}\left(\widehat{\pi}^{h} \widehat{\eta}_{2}\right)+\widehat{D}\left(\widehat{\pi}^{h} \widehat{\eta}_{2}\right) \widehat{\nabla}\left(\widehat{\pi}^{h} \widehat{\eta}_{1}\right)
$$

where for any $z^{h} \in S^{h}$ and $\kappa \in \mathcal{T}^{h}, \widehat{D}\left(\widehat{z}^{h}\right)$ is the $d \times d$ diagonal matrix with diagonal entries

$$
\left[\widehat{D}\left(\widehat{z}^{h}\right)\right]_{i i}:=\frac{1}{2}\left[\widehat{z}^{h}\left(\widehat{p}_{i-1}\right)+\widehat{z}^{h}\left(\widehat{p}_{i}\right)\right] \quad i=1 \rightarrow d .
$$

On combining (2.9), (2.10) and (2.12), we have for all $\eta_{j} \in C(\bar{\Omega})$ that

$$
\nabla\left(\pi^{h}\left[\eta_{1} \eta_{2}\right]\right)=D\left(\pi^{h} \eta_{1}\right) \nabla\left(\pi^{h} \eta_{2}\right)+D\left(\pi^{h} \eta_{2}\right) \nabla\left(\pi^{h} \eta_{1}\right)
$$

where for any $z^{h} \in S^{h}$,

$$
\left.D\left(z^{h}\right)\right|_{\kappa}:=B_{\kappa}^{-T} \widehat{D}\left(\widehat{z}^{h}\right) B_{\kappa}^{T} \quad \forall \kappa \in \mathcal{T}^{h} .
$$

It follows from (2.15) and (2.13) that for all $z^{h} \in S^{h}$ and for all $\kappa \in \mathcal{T}^{h}$

$$
\left\|\left.D\left(z^{h}\right)\right|_{\kappa}\right\|^{2} \leq C\left\|\left.\widehat{D}\left(\widehat{z}^{h}\right)\right|_{\hat{\kappa}}\right\|^{2} \leq C \max _{i=0 \rightarrow d}\left|\widehat{z}^{h}\left(\widehat{p}_{i}\right)\right|^{2}=C \max _{i=0 \rightarrow d}\left|z^{h}\left(p_{i}\right)\right|^{2} \leq C f_{\kappa} \pi^{h}\left[\left(z^{h}\right)^{2}\right],
$$

where $\|\cdot\|$ is the spectral norm on $d \times d$ matrices. Similarly to the above, it follows from (2.15), (2.13) and 
(2.10) that for all $z^{h} \in S^{h}$ and $\kappa \in T^{h}$ that

$$
\left|D\left(z^{h}\right)-z^{h} \mathcal{I}\right|_{0, \infty, \kappa} \leq C\left|\widehat{D}\left(\widehat{z}^{h}\right)-\widehat{z}^{h} \mathcal{I}\right|_{0, \infty, \widehat{\kappa}} \leq C\left|\widehat{\nabla} \widehat{z}^{h}\right|_{0, \infty, \widehat{\kappa}} \leq C h_{\kappa}\left|\nabla z^{h}\right|_{0, \infty, \kappa}
$$

where $\mathcal{I}$ is the $d \times d$ identity matrix.

For any given regularization parameter $\delta \in \mathbb{R}_{>0}$, we then consider the following fully practical finite element approximation of $(\mathrm{P})$ :

$\left(\mathbf{P}_{\delta}^{h, \tau}\right)$ For $n \geq 1$ find $\left\{\Phi_{\delta}^{n}, U_{\delta}^{n}, V_{\delta}^{n}\right\} \in S_{\phi}^{h, n} \times S_{u}^{h} \times S^{h}$ such that $V_{\delta}^{n} \in \pi^{h}\left[\rho\left(U_{\delta}^{n}\right)\right]$ and

$$
\begin{array}{cc}
\left(\sigma_{\delta}^{h}\left(U_{\delta}^{n-1}\right) \nabla \Phi_{\delta}^{n}, \nabla \chi\right)=0 & \forall \chi \in S_{\phi, 0}^{h}, \\
\left(\frac{V_{\delta}^{n}-V_{\delta}^{n-1}}{\tau_{n}}, \chi\right)^{h}+\left(\alpha^{h}\left(U_{\delta}^{n-1}\right) \nabla U_{\delta}^{n}, \nabla \chi\right)+\int_{\Gamma_{N}^{u}} \pi^{h}\left[\gamma\left(U_{\delta}^{n}-\bar{u}_{N}\right) \chi\right] \mathrm{d} s & \forall\left(\sigma_{\delta}^{h}\left(U_{\delta}^{n-1}\right) \nabla \Phi_{\delta}^{n}, D(\chi) \nabla \Phi_{\delta}^{n}\right) \\
& \forall \chi \in S_{u, 0}^{h} ;
\end{array}
$$

where $V_{\delta}^{0} \in S^{h}$ is an approximation to $v^{0}$ and $U_{\delta}^{0}=\pi^{h}\left[\psi\left(V_{\delta}^{0}\right)\right]$ on recalling (1.4).

Remark 2.1. $\left(\mathrm{P}_{\delta}^{h, \tau}\right)$ decouples the updates of the electric potential and the temperature at each time level and is a straightforward finite element approximation of $(\mathrm{P})$, except that $\chi \mathcal{I}$ has been replaced by $D(\chi)$ on the right hand side of $(2.18 \mathrm{~b})$. This choice, which is a simple modification of the standard approximation, enables the discrete analogue of (1.8) to hold; see the proof of Theorem 2.3 below, and in particular the bound (2.29). Although we are not able to establish the bounds in Theorem 2.3 for the standard approximation, in all our practical computations the two approximations lead to numerical results that are graphically indistinguishable, see Section 4.

Below we recall some well-known results concerning $S^{h}$ :

$$
\begin{array}{ll}
\lim _{h \rightarrow 0}\left\|\left(I-\pi^{h}\right) \eta\right\|_{1, q}=0 & \forall \eta \in W^{1, q}(\Omega), \quad q \in(d, \infty] ; \\
\int_{\Omega} \chi^{2} \mathrm{~d} x \leq|\chi|_{h}^{2} \equiv \int_{\Omega} \pi^{h}\left[\chi^{2}\right] \mathrm{d} x \leq(d+2) \int_{\Omega} \chi^{2} \mathrm{~d} x & \forall \chi \in S^{h} ; \\
\int_{\Gamma_{N}^{u}} \chi^{2} \mathrm{~d} s \leq \int_{\Gamma_{N}^{u}} \pi^{h}\left[\chi^{2}\right] \mathrm{d} s \leq(d+1) \int_{\Gamma_{N}^{u}} \chi^{2} \mathrm{~d} s \leq C|\chi|_{1}^{2} & \forall \chi \in S_{u, 0}^{h} ; \\
\left|\left(\chi, z^{h}\right)-\left(\chi, z^{h}\right)^{h}\right| \leq\left|\left(I-\pi^{h}\right)\left(\chi z^{h}\right)\right|_{0,1} \leq C h^{1+m}|\chi|_{m}\left|z^{h}\right|_{1} & \forall z^{h}, \chi \in S^{h}, \quad m=0 \text { or } 1 ; \\
\left|\left(I-\pi^{h}\right)\left(\chi z^{h}\right)\right|_{0,1, \Gamma_{N}^{u}} \leq C h\|\chi\|_{1}\left\|z^{h}\right\|_{1} & \forall z^{h}, \chi \in S^{h} .
\end{array}
$$

Lemma 2.2. Let the assumptions (A2) hold and $U_{\delta}^{n-1} \in S_{u}^{h}, V_{\delta}^{n-1} \in \pi^{h}\left[\rho\left(U_{\delta}^{n-1}\right)\right]$. Then for all $\delta \in(0,1)$ and for all $h, \tau_{n}>0$ there exists a unique solution $\left\{\Phi_{\delta}^{n}, U_{\delta}^{n}, V_{\delta}^{n}\right\}$ to the $n$-th step of $\left(\mathrm{P}_{\delta}^{h, \tau}\right)$ such that

$$
\begin{aligned}
\left(\sigma_{\delta}^{h}\left(U_{\delta}^{n-1}\right) \nabla \Phi_{\delta}^{n}, \nabla \Phi_{\delta}^{n}\right) & \leq\left(\sigma_{\delta}^{h}\left(U_{\delta}^{n-1}\right) \nabla\left[\pi^{h} \bar{\phi}^{n}\right], \nabla\left[\pi^{h} \bar{\phi}^{n}\right]\right) \leq C, \\
m_{\phi} \leq \bar{\phi}_{m}^{n} \leq \Phi_{\delta}^{n}(x) & \leq \bar{\phi}_{M}^{n} \leq M_{\phi} \quad \forall x \in \bar{\Omega} ; \quad \text { where } \bar{\phi}_{m}^{n}:=\bar{\phi}_{m}\left(t_{n}\right) \text { and } \bar{\phi}_{M}^{n}:=\bar{\phi}_{M}\left(t_{n}\right) .
\end{aligned}
$$

Proof. Given $U_{\delta}^{n-1} \in S_{u}^{h}$, it follows immediately from (2.7a) and (2.8) that there exists a unique solution $\Phi_{\delta}^{n} \in S_{\phi}^{h, n}$ to (2.18a). Existence and uniqueness of a solution $\left\{U_{\delta}^{n}, V_{\delta}^{n}\right\} \in S_{u}^{h} \times S^{h}$ to (2.18b) follows on noting that $\rho$ is a maximal monotone operator, see e.g. [3].

The bound (2.24a) follows immediately from choosing $\chi \equiv \Phi_{\delta}^{n}-\pi^{h} \bar{\phi}^{n} \in S_{\phi, 0}^{h}$ in (2.18a), applying a Cauchy-Schwartz inequality and noting (2.19) and (1.2b). Choosing $\chi \equiv \pi^{h}\left[\Phi_{\delta}^{n}-\bar{\phi}_{M}^{n}\right]_{+} \in S_{\phi, 0}^{h}$ in (2.18a), 
on recalling (1.12) and noting (2.4), yields that

$$
\int_{\Omega} \sigma_{\delta}^{h}\left(U_{\delta}^{n-1}\right)\left|\nabla \pi^{h}\left[\Phi_{\delta}^{n}-\bar{\phi}_{M}^{n}\right]_{+}\right|^{2} \leq 0
$$

Combining (2.25) and the fact that $\pi^{h}\left[\Phi_{\delta}^{n}-\bar{\phi}_{M}^{n}\right]_{+} \in S_{\phi, 0}^{h}$ yields that $\pi^{h}\left[\Phi_{\delta}^{n}-\bar{\phi}_{M}^{n}\right]_{+} \equiv 0$ and hence the second inequality in (2.24b). Similarly, choosing $\chi \equiv \pi^{h}\left[\bar{\phi}_{m}^{n}-\Phi_{\delta}^{n}\right]_{+} \in S_{\phi, 0}^{h}$ in (2.18a) yields the first inequality in $(2.24 \mathrm{~b})$.

Throughout this paper, we will assume that the initial data satisfies as $h \rightarrow 0$

$$
S_{u}^{h} \ni U_{\delta}^{0}=\pi^{h}\left[\psi\left(V_{\delta}^{0}\right)\right] \rightarrow u^{0}:=\psi\left(v^{0}\right) \quad \text { strongly in } H^{1}(\Omega), \quad V_{\delta}^{0} \rightarrow v^{0} \quad \text { strongly in } L^{2}(\Omega) .
$$

For example if $v^{0} \in L^{\infty}(\Omega)$ and $u^{0} \in H_{u}^{1}(\Omega) \cap W^{1, r}(\Omega) \subset C(\bar{\Omega}), r>d$, with $u^{0}=0$ on a finite number of curves (surfaces) if $d=2(d=3)$; then on setting $U_{\delta}^{0}=\pi^{h} u^{0}$ and $V_{\delta}^{0}\left(p_{j}\right) \in \rho\left(U_{\delta}^{0}\left(p_{j}\right)\right)$ for all $j \in J$, the first result in (2.26) follows immediately from (2.19) and the second is easily established.

Theorem 2.3. Let the assumptions (A2) hold and $U_{\delta}^{0} \in S_{u}^{h}, V_{\delta}^{0} \in \pi^{h}\left[\rho\left(U_{\delta}^{0}\right)\right]$ satisfy (2.26). Then for all $\delta \in(0,1), h>0$ and for all time partitions $\left\{\tau_{n}\right\}_{n=1}^{N}$ with $\tau_{n} \leq C \tau_{n-1}, n=2 \rightarrow N$, the unique solution $\left\{\Phi_{\delta}^{n}, U_{\delta}^{n}, V_{\delta}^{n}\right\}_{n=1}^{N}$ to $\left(\mathrm{P}_{\delta}^{h, \tau}\right)$ is such that

$$
\max _{n=1 \rightarrow N}\left|V_{\delta}^{n}\right|_{h}^{2}+\max _{n=1 \rightarrow N}\left|U_{\delta}^{n}\right|_{h}^{2}+\sum_{n=1}^{N} \tau_{n}\left|U_{\delta}^{n}\right|_{1}^{2}+\sum_{n=1}^{N} \tau_{n} \int_{\Gamma_{N}^{u}} \pi^{h}\left[\gamma\left(U_{\delta}^{n}\right)^{2}\right] \mathrm{d} s+\sum_{n=1}^{N}\left|U_{\delta}^{n}-U_{\delta}^{n-1}\right|_{h}^{2} \leq C(T) .
$$

Proof. Choosing $\chi \equiv U_{\delta}^{n}-\pi^{h} \bar{u}_{D} \in S_{u, 0}^{h}$ in (2.18b) yields that

$$
\begin{aligned}
& \left(V_{\delta}^{n}-V_{\delta}^{n-1}, U_{\delta}^{n}-\bar{u}_{D}\right)^{h}+\tau_{n}\left(\alpha^{h}\left(U_{\delta}^{n-1}\right) \nabla U_{\delta}^{n}, \nabla\left[U_{\delta}^{n}-\pi^{h} \bar{u}_{D}\right]\right) \\
& \quad+\tau_{n} \int_{\Gamma_{N}^{u}} \pi^{h}\left[\gamma\left(U_{\delta}^{n}-\bar{u}_{N}\right)\left(U_{\delta}^{n}-\bar{u}_{D}\right)\right] \mathrm{d} s=\tau_{n}\left(\sigma_{\delta}^{h}\left(U_{\delta}^{n-1}\right) \nabla \Phi_{\delta}^{n}, D\left(U_{\delta}^{n}-\pi^{h} \bar{u}_{D}\right) \nabla \Phi_{\delta}^{n}\right) .
\end{aligned}
$$

We now apply the discrete analogue of (1.8). On noting (2.14), (2.18a), the fact that $\Phi_{\delta}^{n}-\pi^{h} \bar{\phi}^{n} \in S_{\phi, 0}^{h}$, (2.24a,b), (2.16), (2.20), (2.19), (1.2b) and a Poincaré inequality; it follows for all $\chi \in S_{u, 0}^{h}$ that

$$
\begin{aligned}
\left|\left(\sigma_{\delta}^{h}\left(U_{\delta}^{n-1}\right) \nabla \Phi_{\delta}^{n}, D(\chi) \nabla \Phi_{\delta}^{n}\right)\right| & =\left|\left(\sigma_{\delta}^{h}\left(U_{\delta}^{n-1}\right) \nabla \Phi_{\delta}^{n}, D(\chi) \nabla \pi^{h} \bar{\phi}^{n}-D\left(\Phi_{\delta}^{n}-\pi^{h} \bar{\phi}^{n}\right) \nabla \chi\right)\right| \\
& \leq C\left[|D(\chi)|_{0}\left|\pi^{h} \bar{\phi}^{n}\right|_{1, \infty}+\left|D\left(\Phi_{\delta}^{n}-\pi^{h} \bar{\phi}^{n}\right)\right|_{0, \infty}|\chi|_{1}\right] \leq C|\chi|_{1} .
\end{aligned}
$$

Combining (2.28), (2.29), (2.19) and (1.2a) yields that

$$
\begin{aligned}
\left(V_{\delta}^{n}-V_{\delta}^{n-1}, U_{\delta}^{n}-\bar{u}_{D}\right)^{h}+\frac{1}{2} \tau_{n}\left(\alpha^{h}\left(U_{\delta}^{n-1}\right)\right. & \left.\nabla U_{\delta}^{n}, \nabla U_{\delta}^{n}\right)+\frac{1}{2} \tau_{n} \int_{\Gamma_{N}^{u}} \pi^{h}\left[\gamma\left(U_{\delta}^{n}-\bar{u}_{N}\right)^{2}\right] \mathrm{d} s \\
\leq & C \tau_{n}\left[1+\left|\pi^{h} \bar{u}_{D}\right|_{1}^{2}+\int_{\Gamma_{N}^{u}} \pi^{h}\left[\gamma\left(\bar{u}_{D}-\bar{u}_{N}\right)^{2}\right] \mathrm{d} s\right] \leq C \tau_{n}
\end{aligned}
$$


It follows from the convexity of $\Psi$, recall (1.4), that

$$
\begin{aligned}
\sum_{n=1}^{k}\left(V_{\delta}^{n}-V_{\delta}^{n-1}, U_{\delta}^{n}-\bar{u}_{D}\right)^{h} & =\sum_{n=1}^{k}\left(V_{\delta}^{n}-V_{\delta}^{n-1}, \psi\left(V_{\delta}^{n}\right)-\bar{u}_{D}\right)^{h} \geq \sum_{n=1}^{k}\left(\Psi\left(V_{\delta}^{n}\right)-\Psi\left(V_{\delta}^{n-1}\right), 1\right)^{h}+\left(V_{\delta}^{0}-V_{\delta}^{k}, \bar{u}_{D}\right)^{h} \\
& =\left[\left(\Psi\left(V_{\delta}^{k}\right), 1\right)^{h}-\left(V_{\delta}^{k}, \bar{u}_{D}\right)^{h}\right]-\left[\left(\Psi\left(V_{\delta}^{0}\right), 1\right)^{h}-\left(V_{\delta}^{0}, \bar{u}_{D}\right)^{h}\right] \quad k=1 \rightarrow N .
\end{aligned}
$$

Combining (2.30) and (2.31), and noting (1.2a), (1.4), (2.20) and (2.26), yields that

$$
\begin{aligned}
{\left[\left(\Psi\left(V_{\delta}^{k}\right), 1\right)^{h}-\right.} & \left.\left(V_{\delta}^{k}, \bar{u}_{D}\right)^{h}\right]+\frac{1}{2} \sum_{n=1}^{k} \tau_{n}\left(\alpha^{h}\left(U_{\delta}^{n-1}\right) \nabla U_{\delta}^{n}, \nabla U_{\delta}^{n}\right)+\frac{1}{2} \sum_{n=1}^{k} \tau_{n} \int_{\Gamma_{N}^{u}} \pi^{h}\left[\gamma\left(U_{\delta}^{n}\right)^{2}\right] \mathrm{d} s \\
& \leq C(T)\left[1+\left(\Psi\left(V_{\delta}^{0}\right), 1\right)^{h}-\left(V_{\delta}^{0}, \bar{u}_{D}\right)^{h}\right] \leq C(T)\left[1+\left|V_{\delta}^{0}\right|_{h}^{2}\right] \leq C(T) \quad k=1 \rightarrow N
\end{aligned}
$$

The first four bounds in (2.27) then follow from (2.32) on noting (1.5), (1.2a) and (1.4).

Choosing $\chi \equiv U_{\delta}^{n}-U_{\delta}^{n-1} \in S_{u, 0}^{h}$ in (2.18b), noting the monotonicity of $\rho$, (1.2a), (2.21), (2.29), our time step constraint, bounds 2 and 3 in (2.27) and (2.26), yields that

$$
\begin{aligned}
\sum_{n=1}^{N}\left|U_{\delta}^{n}-U_{\delta}^{n-1}\right|_{h}^{2} & \leq \sum_{n=1}^{N}\left(V_{\delta}^{n}-V_{\delta}^{n-1}, U_{\delta}^{n}-U_{\delta}^{n-1}\right)^{h} \leq C \sum_{n=1}^{N} \tau_{n}\left(a_{n}+1\right)\left|U_{\delta}^{n}-U_{\delta}^{n-1}\right|_{1} \\
& \leq C(T)\left[1+\left(\sum_{n=1}^{N} \tau_{n} a_{n}^{2}\right)^{\frac{1}{2}}\right]\left(\sum_{n=1}^{N} \tau_{n}\left|U_{\delta}^{n}-U_{\delta}^{n-1}\right|_{1}^{2}\right)^{\frac{1}{2}} \leq C(T)
\end{aligned}
$$

where

$$
a_{n}:=\left(\left|U_{\delta}^{n}\right|_{1}^{2}+\int_{\Gamma_{N}^{u}} \pi^{h}\left[\gamma\left(U_{\delta}^{n}\right)^{2}\right] \mathrm{d} s\right)^{\frac{1}{2}} .
$$

Hence the final bound in (2.27) follows immediately from (2.33).

\section{Convergence}

Let

$$
\begin{aligned}
& U_{\delta}(t):=\frac{t-t_{n-1}}{\tau_{n}} U_{\delta}^{n}+\frac{t_{n}-t}{\tau_{n}} U_{\delta}^{n-1} \quad t \in\left[t_{n-1}, t_{n}\right] \quad n \geq 1, \\
& U_{\delta}^{+}(t):=U_{\delta}^{n}, \quad U_{\delta}^{-}(t):=U_{\delta}^{n-1} \quad t \in\left(t_{n-1}, t_{n}\right] \quad n \geq 1 .
\end{aligned}
$$

We note for future reference that

$$
U_{\delta}-U_{\delta}^{ \pm}=\left(t-t_{n}^{ \pm}\right) \frac{\partial U_{\delta}}{\partial t} \quad t \in\left(t_{n-1}, t_{n}\right) \quad n \geq 1,
$$

where $t_{n}^{+}:=t_{n}$ and $t_{n}^{-}:=t_{n-1}$. We introduce also

$$
\bar{\tau}(t):=\tau_{n} \quad t \in\left(t_{n-1}, t_{n}\right] \quad n \geq 1 .
$$

Using the above notation, and introducing analogous notation for $V_{\delta}$ and $\Phi_{\delta},\left(\mathrm{P}_{\delta}^{h, \tau}\right)$ can be restated as: find $\left\{\Phi_{\delta}^{+}, U_{\delta}, V_{\delta}\right\} \in L^{\infty}\left(0, T ; S^{h}\right) \times C\left([0, T] ; S_{u}^{h}\right) \times C\left([0, T] ; S^{h}\right)$ such that $\Phi_{\delta}^{+}\left(\cdot, t_{n}\right) \in S_{\phi}^{h, n}, n=1 \rightarrow N, V_{\delta}^{ \pm} \in$ 
$\pi^{h}\left[\rho\left(U_{\delta}^{ \pm}\right)\right]$and

$$
\begin{aligned}
\int_{0}^{T}\left(\sigma_{\delta}^{h}\left(U_{\delta}^{-}\right) \nabla \Phi_{\delta}^{+}, \nabla \chi\right) \mathrm{d} t=0 & \forall \chi \in L^{2}\left(0, T ; S_{\phi, 0}^{h}\right), \\
\int_{0}^{T}\left[\left(\frac{\partial V_{\delta}}{\partial t}, \chi\right)^{h}+\left(\alpha^{h}\left(U_{\delta}^{-}\right) \nabla U_{\delta}^{+}, \nabla \chi\right)+\int_{\Gamma_{N}^{u}} \pi^{h}\left[\gamma\left(U_{\delta}^{+}-\bar{u}_{N}\right) \chi\right] \mathrm{d} s\right] \mathrm{d} t & \\
=\int_{0}^{T}\left(\sigma_{\delta}^{h}\left(U_{\delta}^{-}\right) \nabla \Phi_{\delta}^{+}, D(\chi) \nabla \Phi_{\delta}^{+}\right) \mathrm{d} t & \forall \chi \in L^{2}\left(0, T ; S_{u, 0}^{h}\right) .
\end{aligned}
$$

Lemma 3.1. Let the assumptions of Theorem 2.3 hold such that $\tau, \delta \rightarrow 0$ as $h \rightarrow 0$. Then there exists a subsequence of $\left\{\Phi_{\delta}^{+}, U_{\delta}, V_{\delta}\right\}_{h}$, where $\left\{\Phi_{\delta}^{+}, U_{\delta}, V_{\delta}\right\}$ solve $\left(\mathrm{P}_{\delta}^{h, \tau}\right)$, and functions

$$
\phi \in L^{\infty}\left(\Omega_{T}\right), \quad u \in L^{\infty}\left(0, T ; L^{2}(\Omega)\right) \cap L^{2}\left(0, T ; H_{u}^{1}(\Omega)\right), \quad v \in L^{\infty}\left(0, T ; L^{2}(\Omega)\right)
$$

and $g \in L^{2}\left(\Omega_{T}\right)$ such that $v \in \rho(u)$ for a.e. $(x, t) \in \Omega_{T}$ and as $h \rightarrow 0$

$$
\begin{aligned}
& \Phi_{\delta}^{+} \rightarrow \phi \quad \text { weak-* in } L^{\infty}\left(\Omega_{T}\right), \\
& {\left[\sigma_{\delta}^{h}\left(U_{\delta}^{-}\right)\right]^{\frac{1}{2}} \nabla \Phi_{\delta}^{+} \rightarrow g \quad \text { weakly in } L^{2}\left(\Omega_{T}\right) \text {, }} \\
& U_{\delta}, U_{\delta}^{ \pm} \rightarrow u \quad \text { weak-* in } L^{\infty}\left(0, T ; L^{2}(\Omega)\right) \text {, } \\
& U_{\delta}, U_{\delta}^{ \pm} \rightarrow u \quad \text { weakly in } L^{2}\left(0, T ; H^{1}(\Omega)\right) \text {, } \\
& U_{\delta}, U_{\delta}^{ \pm} \rightarrow u \quad \text { weakly in } L^{2}\left(0, T ; L^{2}(\partial \Omega)\right) \text {, } \\
& V_{\delta} \rightarrow v \quad \text { weak-* in } L^{\infty}\left(0, T ; L^{2}(\Omega)\right) .
\end{aligned}
$$

If in addition $\tau_{n}=\tau, n=1 \rightarrow N(h)$, then as $h \rightarrow 0$

$$
\begin{aligned}
& U_{\delta}, U_{\delta}^{ \pm} \rightarrow u \quad \text { strongly in } L^{2}\left(0, T ; L^{q}(\Omega)\right), \quad q \in[1, s), \\
& \sigma_{\delta}^{h}\left(U_{\delta}^{-}\right) \rightarrow \sigma(u) \quad \text { strongly in } L^{2}\left(0, T ; L^{q}(\Omega)\right), \quad q \in[1, s), \\
& {\left[\sigma_{\delta}^{h}\left(U_{\delta}^{-}\right)\right]^{\frac{1}{2}} \rightarrow[\sigma(u)]^{\frac{1}{2}} \quad \text { strongly in } L^{2}\left(0, T ; L^{q}(\Omega)\right), \quad q \in[1, s) \text {, }} \\
& \alpha^{h}\left(U_{\delta}^{-}\right) \rightarrow \alpha(u) \quad \text { strongly in } L^{2}\left(0, T ; L^{q}(\Omega)\right), \quad q \in[1, s) \text {; }
\end{aligned}
$$

where $s=\infty$ if $d=2$ and $s=6$ if $d=3$.

Proof. Noting the definitions $(3.1 \mathrm{a}, \mathrm{b}),(3.3)$, the bounds $(2.24 \mathrm{a}, \mathrm{b})$ and $(2.27)$ imply that

$$
\begin{aligned}
&\left\|\Phi_{\delta}^{+}\right\|_{L^{\infty}\left(\Omega_{T}\right)}^{2}+\left\|\left[\sigma_{\delta}^{h}\left(U_{\delta}^{-}\right)\right]^{\frac{1}{2}} \nabla \Phi_{\delta}^{+}\right\|_{L^{2}\left(\Omega_{T}\right)}^{2}+\left\|V_{\delta}^{( \pm)}\right\|_{L^{\infty}\left(0, T ; L^{2}(\Omega)\right)}^{2} \\
&+\left\|U_{\delta}^{( \pm)}\right\|_{L^{\infty}\left(0, T ; L^{2}(\Omega)\right)}^{2}+\left\|U_{\delta}^{( \pm)}\right\|_{L^{2}\left(0, T ; H^{1}(\Omega)\right)}^{2}+\left\|\bar{\tau}^{\frac{1}{2}} \frac{\partial U_{\delta}}{\partial t}\right\|_{L^{2}\left(\Omega_{T}\right)}^{2} \leq C(T) .
\end{aligned}
$$

Furthermore, we deduce from (3.2) and (3.8) that

$$
\left\|U_{\delta}-U_{\delta}^{ \pm}\right\|_{L^{2}\left(\Omega_{T}\right)}^{2} \leq\left\|\bar{\tau} \frac{\partial U_{\delta}}{\partial t}\right\|_{L^{2}\left(\Omega_{T}\right)}^{2} \leq C(T) \tau .
$$

Hence on noting (3.8), (3.9), (2.19) and (1.2a) we can choose a subsequence $\left\{\Phi_{\delta}^{+}, U_{\delta}, V_{\delta}\right\}_{h}$ such that the convergence results $(3.5)$ and $(3.6 \mathrm{a}-\mathrm{f})$ hold. 
In the next part of the proof we will establish the strong convergence results $(3.7 \mathrm{a}, \mathrm{b})$. It follows from $(2.18 \mathrm{~b})$ for $m=0 \rightarrow N-\ell, \ell \in\{1, \ldots, N\}$ fixed, that

$$
\begin{aligned}
& \sum_{n=m+1}^{m+\ell} \tau_{n}\left(\frac{V_{\delta}^{n}-V_{\delta}^{n-1}}{\tau_{n}}, U_{\delta}^{m+\ell}-U_{\delta}^{m}\right)^{h}=-\sum_{n=m+1}^{m+\ell} \tau_{n}\left[\left(\alpha^{h}\left(U_{\delta}^{n-1}\right) \nabla U_{\delta}^{n}, \nabla\left[U_{\delta}^{m+\ell}-U_{\delta}^{m}\right]\right)\right. \\
& \left.\quad+\int_{\Gamma_{N}^{u}} \pi^{h}\left[\gamma\left(U_{\delta}^{n}-\bar{u}_{N}\right)\left(U_{\delta}^{m+\ell}-U_{\delta}^{m}\right)\right] \mathrm{d} s-\left(\sigma_{\delta}^{h}\left(U_{\delta}^{n-1}\right) \Phi_{\delta}^{n}, D\left(U_{\delta}^{m+\ell}-U_{\delta}^{m}\right) \nabla \Phi_{\delta}^{n},\right)\right] .
\end{aligned}
$$

Similarly to (2.33), we obtain from (3.10), on noting the monotonicity of $\rho,(1.2 \mathrm{a}),(2.21),(2.29)$ and $(2.34)$, that

$$
\begin{aligned}
\left|U_{\delta}^{m+\ell}-U_{\delta}^{m}\right|_{h}^{2} & \leq\left(V_{\delta}^{m+\ell}-V_{\delta}^{m}, U_{\delta}^{m+\ell}-U_{\delta}^{m}\right)^{h} \leq C \sum_{n=m+1}^{m+\ell} \tau_{n}\left(a_{n}+1\right)\left|U_{\delta}^{m+\ell}-U_{\delta}^{m}\right|_{1} \\
& =C \sum_{k=1}^{\ell} \tau_{m+k}\left(a_{m+k}+1\right)\left|U_{\delta}^{m+\ell}-U_{\delta}^{m}\right|_{1} .
\end{aligned}
$$

Summing (3.11) for $m=0 \rightarrow N-\ell$ yields, on noting the uniform time step assumption, (2.27) and (2.26), that

$$
\begin{aligned}
\sum_{m=0}^{N-\ell} \tau\left|U_{\delta}^{m+\ell}-U_{\delta}^{m}\right|_{h}^{2} & \leq C \sum_{k=1}^{\ell} \tau \sum_{m=0}^{N-\ell} \tau a_{m+k}\left|U_{\delta}^{m+\ell}-U_{\delta}^{m}\right|_{1} \\
& \leq C \sum_{k=1}^{\ell} \tau\left[\sum_{m=0}^{N-\ell} \tau a_{m+k}^{2}\right]^{\frac{1}{2}}\left[\sum_{m=0}^{N-\ell} \tau\left|U_{\delta}^{m+\ell}-U_{\delta}^{m}\right|_{1}^{2}\right]^{\frac{1}{2}} \leq C(T) \ell \tau
\end{aligned}
$$

Combining (3.12), (2.2), (2.20) and (3.1b) yields that

$$
\int_{0}^{T-\theta}\left|U_{\delta}^{ \pm}(t+\theta)-U_{\delta}^{ \pm}(t)\right|_{0}^{2} \mathrm{~d} t \leq C(T) \theta
$$

for $\theta=\ell \tau$. It is a simple matter to generalise (3.13) to arbitrary $\theta \in(0, T)$ with $\theta=\mu \tau, \mu \in(0, N)$; see e.g. [2], Lemma 3.2. This yields (3.13) for all $\theta \in(0, T)$. It follows from this and (3.6d), on noting (1.10), that (3.7a) holds.

Furthermore, we have from (1.6) that for $q \in[2, s)$

$$
\left\|\sigma_{\delta}^{h}\left(U_{\delta}^{-}\right)-\sigma(u)\right\|_{L^{2}\left(0, T ; L^{q}(\Omega)\right)} \leq\left\|\sigma_{\delta}^{h}\left(U_{\delta}^{-}\right)-\sigma\left(U_{\delta}^{-}\right)\right\|_{L^{2}\left(0, T ; L^{q}(\Omega)\right)}+C\left\|U_{\delta}^{-}-u\right\|_{L^{2}\left(0, T ; L^{q}(\Omega)\right)} .
$$

It follows from (2.8), (2.5), an inverse inequality and (2.4) that for all $\kappa \in \mathcal{T}^{h}$ and $t \in(0, T)$

$$
\begin{aligned}
\left\|\sigma_{\delta}^{h}\left(U_{\delta}^{-}\right)-\sigma\left(U_{\delta}^{-}\right)\right\|_{0, q, \kappa}^{q} & \leq C\left[\delta+h_{\kappa}^{q}\left|\nabla\left(\pi^{h}\left[\sigma\left(U_{\delta}^{-}\right)\right]\right)\right|_{0, q, \kappa}^{q}\right] \\
& \leq C\left[\delta+h_{\kappa}^{2}\left|\pi^{h}\left[\sigma\left(U_{\delta}^{-}\right)\right]\right|_{0, \infty, \kappa}^{q-2}\left|\nabla\left(\pi^{h}\left[\sigma\left(U_{\delta}^{-}\right)\right]\right)\right|_{0, \kappa}^{2}\right] \leq C\left[\delta+h_{\kappa}^{2}\left|\nabla U_{\delta}^{-}\right|_{0, \kappa}^{2}\right] .
\end{aligned}
$$

From (3.15) and (3.8) we have that

$$
\left\|\sigma_{\delta}^{h}\left(U_{\delta}^{-}\right)-\sigma\left(U_{\delta}^{-}\right)\right\|_{L^{2}\left(0, T ; L^{q}(\Omega)\right)} \leq C(T)\left[\delta+h^{\frac{2}{q}}\left\|U_{\delta}^{-}\right\|_{L^{2}\left(0, T ; H^{1}(\Omega)\right)}^{\frac{2}{q}}\right] \leq C(T)\left[\delta+h^{\frac{2}{q}}\right] .
$$


Combining (3.14) and (3.16), and noting (3.7a) and our assumption on $\delta$, yields the desired result (3.7b). The result $(3.7 \mathrm{c})$ follows immediately from $(3.7 \mathrm{~b})$, and the result (3.7d) follows similarly to $(3.7 \mathrm{~b})$.

It remains to be shown that $v \in \rho(u)$ for a.e. $(x, t) \in \Omega_{T}$. It follows from the monotonicity of $\rho$ and as $U_{\delta}=\pi^{h}\left[\psi\left(V_{\delta}\right)\right]$ that

$$
\int_{\Omega_{T}}\left(\rho(\eta)-V_{\delta}\right)\left[\left(\eta-U_{\delta}\right)+\left(\pi^{h}-I\right) \psi\left(V_{\delta}\right)\right] \mathrm{d} x \mathrm{~d} t \geq 0 \quad \forall \eta \in L^{2}\left(\Omega_{T}\right) .
$$

Furthermore, it follows from (2.5) that

$$
\left\|\left(\pi^{h}-I\right) \psi\left(V_{\delta}\right)\right\|_{L^{2}\left(\Omega_{T}\right)} \leq C h\left\|U_{\delta}\right\|_{L^{2}\left(0, T ; H^{1}(\Omega)\right)} .
$$

Combining (3.17) and (3.18), on noting (3.8), (3.7a) and (3.6f), yields that as $h \rightarrow 0$

$$
\int_{\Omega_{T}}(\rho(\eta)-v)(\eta-u) \mathrm{d} x \mathrm{~d} t \geq 0 \quad \forall \eta \in L^{2}\left(\Omega_{T}\right)
$$

and hence that $v \in \rho(u)$ for a.e. $(x, t) \in \Omega_{T}$ due to the monotonicity of $\rho$.

We now adapt the arguments in $[9,10]$ in order to show that $\{\phi, u, v\}$ is indeed a weak solution of $(\mathrm{P})$, $(1.1 \mathrm{a}-\mathrm{e})$.

Let $\mathcal{A}$ be defined by

$$
\mathcal{A}:=\left\{f \in C^{0,1}(\mathbb{R}): \quad \sup _{s \in \mathbb{R}}|f(s)|<\infty \quad \text { and } \quad \inf \{s \in \mathbb{R}: f(s) \neq 0\}>0\right\} .
$$

Furthermore, for any $w \in L^{2}\left(0, T ; H^{1}(\Omega)\right)$ let $X_{w} \subset L^{\infty}\left(\Omega_{T}\right)$ be defined by

$$
X_{w}:=\left\{\varphi \in L^{\infty}\left(\Omega_{T}\right): f(w) \varphi \in L^{2}\left(0, T ; H^{1}(\Omega)\right) \quad \forall f \in \mathcal{A}\right\} .
$$

Lemma 3.2. Let the assumptions of Lemma 3.1 hold. It follows that the subsequence $\left\{\Phi_{\delta}^{+}, U_{\delta}, V_{\delta}\right\}_{h}$ in Lemma 3.1 is such that for all $f \in \mathcal{A}$

$$
f\left(U_{\delta}^{-}\right) \Phi_{\delta}^{+} \rightarrow f(u) \phi \quad \text { weakly in } L^{2}\left(0, T ; H^{1}(\Omega)\right) \quad \text { as } h \rightarrow 0 .
$$

In particular, $\phi \in X_{u}$.

Proof. Let $f \in \mathcal{A}$ with Lipschitz constant $L_{f}, \sup _{s \in \mathbb{R}}|f(s)| \leq M_{f}$ and $c_{f}:=\inf \{s \in \mathbb{R}: f(s) \neq 0\}>0$. We have, on noting (3.7a), that

$$
\left\|f\left(U_{\delta}^{-}\right)-f(u)\right\|_{L^{2}\left(\Omega_{T}\right)} \leq L_{f}\left\|U_{\delta}^{-}-u\right\|_{L^{2}\left(\Omega_{T}\right)} \rightarrow 0 \quad \text { as } h \rightarrow 0 .
$$

Combining (3.22) and (3.6a) yields that

$$
f\left(U_{\delta}^{-}\right) \Phi_{\delta}^{+} \rightarrow f(u) \phi \quad \text { weakly in } L^{2}\left(\Omega_{T}\right) \quad \text { as } h \rightarrow 0 .
$$

It follows from (3.8) that

$$
\begin{aligned}
\left\|\nabla\left[f\left(U_{\delta}^{-}\right) \Phi_{\delta}^{+}\right]\right\|_{L^{2}\left(\Omega_{T}\right)} & \leq\left\|\nabla\left[f\left(U_{\delta}^{-}\right)\right] \Phi_{\delta}^{+}\right\|_{L^{2}\left(\Omega_{T}\right)}+\left\|f\left(U_{\delta}^{-}\right) \nabla \Phi_{\delta}^{+}\right\|_{L^{2}\left(\Omega_{T}\right)} \\
& \leq L_{f}\left\|U_{\delta}^{-}\right\|_{L^{2}\left(0, T ; H^{1}(\Omega)\right)}\left\|\Phi_{\delta}^{+}\right\|_{L^{\infty}\left(\Omega_{T}\right)}+M_{f}\left(\int_{U_{\delta}^{-}>c_{f}}\left|\nabla \Phi_{\delta}^{+}\right|^{2} \mathrm{~d} x \mathrm{~d} t\right)^{\frac{1}{2}} \\
& \leq C\left(M_{f}, L_{f}, T\right)\left[1+\int_{U_{\delta}^{-}>c_{f}}\left|\nabla \Phi_{\delta}^{+}\right|^{2} \mathrm{~d} x \mathrm{~d} t\right]^{\frac{1}{2}} .
\end{aligned}
$$


Moreover, if $U_{\delta}^{-}(x)>c_{f}$ for some $x \in \bar{\kappa}$, then there exists a $j \in J$ such that $p_{j}$ is a vertex of $\kappa$ and $U_{\delta}^{-}\left(p_{j}\right)>c_{f}$. Hence it holds that

$$
\left.\sigma_{\delta}^{h}\left(U_{\delta}^{-}\right)\right|_{\kappa}:=f_{\kappa} \pi^{h}\left[\sigma_{\delta}\left(U_{\delta}^{-}\right)\right]>\frac{1}{d+1} \sigma_{\delta}\left(c_{f}\right)>\frac{1}{d+1} \sigma\left(c_{f}\right)>0 .
$$

Combining (3.24), (3.25) and (3.8) yields that

$$
\left\|f\left(U_{\delta}^{-}\right) \Phi_{\delta}^{+}\right\|_{L^{2}\left(0, T ; H^{1}(\Omega)\right)} \leq C\left(c_{f}, M_{f}, L_{f}, T\right) .
$$

The desired result (3.21) then follows from (3.23) and (3.26). Finally, on noting (3.5), (3.20) and (3.21), we have that $\phi \in X_{u}$.

Lemma 3.3. Let the assumptions of Lemma 3.1 hold. Then the limit $g$ in (3.6b) is such that

$$
g=[\sigma(u)]^{\frac{1}{2}} \nabla \phi \quad \text { a.e. on } P_{u}:=\left\{(x, t) \in \bar{\Omega}_{T}: u(x, t)>0\right\} .
$$

Proof. The proof is similar to the proof in [9], Lemma 3.4. It follows from (3.6d), (3.7a) and (1.11a,b) that given $\varepsilon>0$, there exists a $\vartheta_{\varepsilon} \in L^{1}\left(0, T ; W^{1,1}(\Omega)\right)$ satisfying $(1.11 \mathrm{~b})$, with $s=1$, and a further subsequence of the subsequence $\left\{\Phi_{\delta}^{+}, U_{\delta}, V_{\delta}\right\}_{h}$ in Lemma 3.1 such that

$$
U_{\delta}^{-} \rightarrow u \quad \text { uniformly on }\left\{\vartheta_{\varepsilon}>0\right\} \quad \text { as } h \rightarrow 0 \text {. }
$$

Now fix $f \in \mathcal{A}$ with $c_{f}:=\inf \{s \in \mathbb{R}: f(s) \neq 0\}>0$. It follows immediately from (3.28) that, for $h$ sufficiently small,

$$
U_{\delta}^{-} \geq \frac{1}{2} c_{f} \quad \text { on }\left\{\vartheta_{\varepsilon}>0\right\} \cap\left\{u>c_{f}\right\}
$$

Subsequently, we choose $f_{1} \in \mathcal{A}$ such that $f_{1}=1$ on $\left[\frac{1}{2} c_{f}, \infty\right)$. Now $(3.6 \mathrm{~b})$ combined with $(3.7 \mathrm{c})$, Lemma 3.2 , $[\sigma(u)]^{\frac{1}{2}} \vartheta_{\varepsilon} f(u) \in L^{\infty}\left(\Omega_{T}\right)$ and a density argument implies that

$$
\begin{aligned}
g \vartheta_{\varepsilon} f(u) \leftarrow\left[\sigma_{\delta}^{h}\left(U_{\delta}^{-}\right)\right]^{\frac{1}{2}} \nabla \Phi_{\delta}^{+} \vartheta_{\varepsilon} f(u) & =\left[\sigma_{\delta}^{h}\left(U_{\delta}^{-}\right)\right]^{\frac{1}{2}} \nabla\left(f_{1}\left(U_{\delta}^{-}\right) \Phi_{\delta}^{+}\right) \vartheta_{\varepsilon} f(u) \\
& \rightarrow[\sigma(u)]^{\frac{1}{2}} \nabla\left(f_{1}(u) \phi\right) \vartheta_{\varepsilon} f(u)=[\sigma(u)]^{\frac{1}{2}} \nabla \phi \vartheta_{\varepsilon} f(u) \quad \text { weakly in } L^{2}\left(\Omega_{T}\right)
\end{aligned}
$$

Repeating the above for any $f \in \mathcal{A}$, we have that

$$
g \vartheta_{\varepsilon} f(u)=[\sigma(u)]^{\frac{1}{2}} \nabla \phi \vartheta_{\varepsilon} f(u) \quad \text { a.e. on } \Omega_{T} \quad \forall f \in \mathcal{A} \text {. }
$$

Now letting $\varepsilon \rightarrow 0$, and noting (1.11b), yields that

$$
g f(u)=[\sigma(u)]^{\frac{1}{2}} \nabla \phi f(u) \quad \text { a.e. on } \Omega_{T} \quad \forall f \in \mathcal{A} \text {. }
$$

This implies the desired result (3.27).

With just the weak convergence (3.6b); it is not possible to pass to the limit $h \rightarrow 0$ on the right hand side of $(2.18 \mathrm{~b})$, and hence prove convergence of $\left(\mathrm{P}_{\delta}^{h, \tau}\right)$ to $(\mathrm{P})$. Therefore the following lemma plays a crucial role.

Lemma 3.4. Let the assumptions of Lemma 3.1 hold. Then the subsequence $\left\{\Phi_{\delta}^{+}, U_{\delta}, V_{\delta}\right\}_{h}$ in Lemma 3.1 is such that

$$
\left[\sigma_{\delta}^{h}\left(U_{\delta}^{-}\right)\right]^{\frac{1}{2}} \nabla \Phi_{\delta}^{+} \rightarrow g \quad \text { strongly in } L^{2}\left(\Omega_{T}\right) \quad \text { as } h \rightarrow 0, \quad \text { and } \quad g=0 \text { on } \Omega_{T} \backslash P_{u} .
$$


Proof. The proof is similar to the proof in [10], Lemma 2.5. Choosing $\chi \equiv \Phi_{\delta}^{+}-\pi^{h} \bar{\phi}^{+}$in (3.4a) yields, on noting (3.7c), (3.6b), (2.19), (1.2b), the notation (3.1a,b), $\tau \rightarrow 0$ as $h \rightarrow 0,(3.27)$ and as $\sigma(u)=0$ on $\Omega_{T} \backslash P_{u}$, that

$$
\begin{aligned}
\int_{\Omega_{T}} \sigma_{\delta}^{h}\left(U_{\delta}^{-}\right)\left|\nabla \Phi_{\delta}^{+}\right|^{2} \mathrm{~d} x \mathrm{~d} t & =\int_{\Omega_{T}} \sigma_{\delta}^{h}\left(U_{\delta}^{-}\right) \nabla \Phi_{\delta}^{+} \cdot \nabla\left[\pi^{h} \bar{\phi}^{+}\right] \mathrm{d} x \mathrm{~d} t \\
& \rightarrow \int_{\Omega_{T}}[\sigma(u)]^{\frac{1}{2}} g \cdot \nabla \bar{\phi} \mathrm{d} x \mathrm{~d} t=\int_{P_{u}} \sigma(u) \nabla \phi . \nabla \bar{\phi} \mathrm{d} x \mathrm{~d} t \quad \text { as } h \rightarrow 0 .
\end{aligned}
$$

Similarly, it follows on choosing $\pi^{h} \eta^{\star}$ in (3.4a), where $\eta^{\star} \in L^{\infty}\left(0, T ; H_{\phi, 0}^{1}(\Omega) \cap W^{1, \infty}(\Omega)\right)$, and noting (3.7c), (3.6b), (2.19), (3.27), a density argument and $\sigma(u)=0$ on $\Omega_{T} \backslash P_{u}$, that

$$
\int_{\Omega_{T}}[\sigma(u)]^{\frac{1}{2}} g \cdot \nabla \eta \mathrm{d} x \mathrm{~d} t=\int_{P_{u}} \sigma(u) \nabla \phi . \nabla \eta \mathrm{d} x \mathrm{~d} t=0 \quad \forall \eta \in L^{2}\left(0, T ; H_{\phi, 0}^{1}(\Omega)\right) .
$$

Next, we observe on noting (1.6) that

$$
\int_{0}^{1}[\sigma(q)]^{-\frac{1}{2}} \mathrm{~d} q=\infty \quad \Longrightarrow \quad \forall \varepsilon>0, \exists \text { a unique } \mu(\varepsilon) \in(0, \varepsilon) \text { s.t. } \int_{\mu}^{\varepsilon}[\sigma(q)]^{-\frac{1}{2}} \mathrm{~d} q=1 .
$$

Let $f_{\mu, \varepsilon} \in \mathcal{A}$ be defined by

$$
f_{\mu, \varepsilon}(s):=\left\{\begin{array}{ll}
1 & \text { if } s \geq \varepsilon, \\
\int_{\mu}^{s}[\sigma(q)]^{-\frac{1}{2}} \mathrm{~d} q & \text { if } s \in[\mu, \varepsilon], \\
0 & \text { if } s \leq \mu ;
\end{array} \quad \Longrightarrow \quad f_{\mu, \varepsilon}^{\prime}(s):= \begin{cases}0 & \text { if } s>\varepsilon, \\
{[\sigma(s)]^{-\frac{1}{2}}} & \text { if } s \in(\mu, \varepsilon), \\
0 & \text { if } s<\mu .\end{cases}\right.
$$

Choosing $\eta \equiv f_{\mu, \varepsilon}(u)(\phi-\bar{\phi})$ in (3.31) and recalling (3.33), we obtain that

$$
\begin{aligned}
\int_{P_{u}} \sigma(u) \nabla \phi \cdot \nabla(\phi-\bar{\phi}) \mathrm{d} x \mathrm{~d} t & =\lim _{\varepsilon \rightarrow 0} \int_{P_{u}} \sigma(u) \nabla \phi \cdot \nabla(\phi-\bar{\phi}) f_{\mu, \varepsilon}(u) \mathrm{d} x \mathrm{~d} t \\
& =-\lim _{\varepsilon \rightarrow 0} \int_{\{\mu<u<\varepsilon\} \cap P_{u}}[\sigma(u)]^{\frac{1}{2}} \nabla \phi \cdot \nabla u(\phi-\bar{\phi}) \mathrm{d} x \mathrm{~d} t=0 .
\end{aligned}
$$

Combining (3.6b), (3.30) and (3.34) yields that

$$
\int_{\Omega_{T}}|g|^{2} \mathrm{~d} x \mathrm{~d} t \leq \lim _{h \rightarrow 0} \int_{\Omega_{T}} \sigma_{\delta}^{h}\left(U_{\delta}^{-}\right)\left|\nabla \Phi_{\delta}^{+}\right|^{2} \mathrm{~d} x \mathrm{~d} t=\int_{P_{u}} \sigma(u)|\nabla \phi|^{2} \mathrm{~d} x \mathrm{~d} t .
$$

This together with (3.27) implies that $g=0$ on $\Omega_{T} \backslash P_{u}$. Therefore it follows from this, (3.35), (3.6b) and (3.27) that

$$
\int_{\Omega_{T}}\left|\left[\sigma_{\delta}^{h}\left(U_{\delta}^{-}\right)\right]^{\frac{1}{2}} \nabla \Phi_{\delta}^{+}-g\right|^{2} \mathrm{~d} x \mathrm{~d} t \rightarrow 0 \quad \text { as } h \rightarrow 0,
$$

and hence the desired result (3.29).

Theorem 3.5. Let the assumptions of Lemma 3.1 hold. Then there exists a subsequence of $\left\{\Phi_{\delta}^{+}, U_{\delta}, V_{\delta}\right\}_{h}$, where $\left\{\Phi_{\delta}^{+}, U_{\delta}, V_{\delta}\right\}$ solve $\left(\mathrm{P}_{\delta}^{h, \tau}\right)$, and functions $\{\phi, u, v\}$ satisfying (3.5) such that as $h \rightarrow 0$ the following hold: (3.6a-f), (3.7a-d), (3.29) and (3.27). Furthermore, we have that $\{\phi, u, v\}$ fulfil $\phi \in X_{u}, \phi=\bar{\phi}$ on $\left(\Gamma_{D}^{\phi} \cap P_{u}\right) \times(0, T]$, 
$v \in \rho(u)$ for a.e. $(x, t) \in \Omega_{T} ;$ and they satisfy

$$
\begin{aligned}
& \int_{P_{u}} \sigma(u) \nabla \phi \cdot \nabla \eta \mathrm{d} x \mathrm{~d} t=0 \\
& -\int_{\Omega_{T}} v \frac{\partial \eta}{\partial t} \mathrm{~d} x \mathrm{~d} t-\int_{\Omega} v^{0} \eta(x, 0) \mathrm{d} x+\int_{\Omega_{T}} \alpha(u) \nabla u . \nabla \eta \mathrm{d} x \mathrm{~d} t+\int_{0}^{T} \int_{\Gamma_{N}^{u}} \gamma\left(u-\bar{u}_{N}\right) \eta \mathrm{d} s \mathrm{~d} t \\
& \left.\quad=\int_{P_{u}} \sigma(u)|\nabla \phi|^{2} \eta \mathrm{d} x \mathrm{~d} t \quad \forall \eta \in H_{\phi, 0}^{1}(\Omega)\right),(3.36 \mathrm{a})
\end{aligned}
$$

Proof. The desired result (3.36a) follows immediately from (3.31). Noting (3.21), (3.8), (3.7a) and (2.19) we have for $f \in \mathcal{A}$, recall (3.19), that as $h \rightarrow 0$

$$
f\left(U_{\delta}^{-}\right)\left[\Phi_{\delta}^{+}-\pi^{h} \bar{\phi}^{+}\right] \rightarrow f(u)[\phi-\bar{\phi}] \quad \text { weakly in } L^{2}\left(0, T ; H^{1}(\Omega)\right) \quad \Longrightarrow \quad \text { weakly in } L^{2}\left(0, T ; L^{2}(\partial \Omega)\right)
$$

As $\Phi_{\delta}^{+}=\pi^{h} \bar{\phi}^{+}$on $\Gamma_{D}^{\phi}$ and $\tau \rightarrow 0$ as $h \rightarrow 0$, it follows from (3.37) that $\phi=\bar{\phi}$ on $\left(\Gamma_{D}^{\phi} \cap P_{u}\right) \times(0, T]$.

We now consider (3.36b). For any $\eta \in H^{1}\left(0, T ; H_{u, 0}^{1}(\Omega) \cap W^{1, \infty}(\Omega)\right)$ with $\eta(\cdot, T)=0$, we choose $\chi \equiv \pi^{h} \eta$ in (3.4b) and now analyse the subsequent terms. Firstly $(2.22)$, the embedding $H^{1}(0, T ; X) \hookrightarrow C([0, T] ; X)$, (3.8) and (2.19) yield that

$$
\begin{aligned}
\left|\int_{0}^{T}\left[\left(\frac{\partial V_{\delta}}{\partial t}, \pi^{h} \eta\right)^{h}+\left(V_{\delta}, \frac{\partial\left(\pi^{h} \eta\right)}{\partial t}\right)\right] \mathrm{d} t+\left(V_{\delta}(\cdot, 0), \pi^{h} \eta(\cdot, 0)\right)\right| \\
\leq\left|\int_{0}^{T}\left[\left(V_{\delta}, \frac{\partial\left(\pi^{h} \eta\right)}{\partial t}\right)-\left(V_{\delta}, \frac{\partial\left(\pi^{h} \eta\right)}{\partial t}\right)^{h}\right] \mathrm{d} t\right|+\left|\left(V_{\delta}(\cdot, 0), \pi^{h} \eta(\cdot, 0)\right)-\left(V_{\delta}(\cdot, 0), \pi^{h} \eta(\cdot, 0)\right)^{h}\right| \\
\leq C h\left\|V_{\delta}\right\|_{L^{\infty}\left(0, T ; L^{2}(\Omega)\right)}\left\|\pi^{h} \eta\right\|_{H^{1}\left(0, T ; H^{1}(\Omega)\right)} \leq C h\|\eta\|_{H^{1}\left(0, T ; W^{1, \infty}(\Omega)\right)}
\end{aligned}
$$

Furthermore, it follows from the embedding $H^{1}(0, T ; X) \hookrightarrow C([0, T] ; X)$, and (3.8) that

$$
\begin{aligned}
& \mid \int_{0}^{T}\left(V_{\delta}, \frac{\partial\left(\left(I-\pi^{h}\right) \eta\right)}{\partial t}\right) \mathrm{d} t|+|\left(V_{\delta}(\cdot, 0),\left(I-\pi^{h}\right) \eta(\cdot, 0)\right) \mid \\
& \leq C\left\|V_{\delta}\right\|_{L^{\infty}\left(0, T ; L^{2}(\Omega)\right)}\left\|\left(I-\pi^{h}\right) \eta\right\|_{H^{1}\left(0, T ; H^{1}(\Omega)\right)} \leq C\left\|\left(I-\pi^{h}\right) \eta\right\|_{H^{1}\left(0, T ; H^{1}(\Omega)\right)}
\end{aligned}
$$

Combining (3.38), (3.39), (2.19) and (3.6f) yields that

$$
\int_{0}^{T}\left(\frac{\partial V_{\delta}}{\partial t}, \pi^{h} \eta\right)^{h} \mathrm{~d} t \rightarrow-\int_{0}^{T}\left(v, \frac{\partial \eta}{\partial t}\right) \mathrm{d} t-\left(v^{0}, \eta(\cdot, 0)\right) \quad \text { as } h \rightarrow 0
$$

Moreover, it holds on noting (2.23), (2.19), (1.2a), (2.14), (2.16), (2.20) and (3.8) that

$$
\begin{aligned}
& \left|\int_{0}^{T} \int_{\Gamma_{N}^{u}}\left(\pi^{h}\left[\gamma\left(U_{\delta}^{+}-\bar{u}_{N}\right) \eta\right]-\left(\pi^{h} \gamma\right)\left(U_{\delta}^{+}-\pi^{h} \bar{u}_{N}\right) \pi^{h} \eta\right) \mathrm{d} s \mathrm{~d} t\right| \\
& \leq C h\left[\left\|\pi^{h} \gamma\right\|_{1}\left\|\pi^{h}\left[\left(U_{\delta}^{+}-\bar{u}_{N}\right) \eta\right]\right\|\left\|_{L^{1}\left(0, T ; H^{1}(\Omega)\right)}+\left|\pi^{h} \gamma\right|_{0, \infty}\right\| U_{\delta}^{+}-\pi^{h} \bar{u}_{N}\left\|_{L^{2}\left(0, T ; H^{1}(\Omega)\right)}\right\| \pi^{h} \eta \|_{L^{2}\left(0, T ; H^{1}(\Omega)\right)}\right] \\
& \leq C h\|\gamma\|_{1, r}\left\|U_{\delta}^{+}-\pi^{h} \bar{u}_{N}\right\|_{L^{2}\left(0, T ; H^{1}(\Omega)\right)}\left\|\pi^{h} \eta\right\|_{L^{2}\left(0, T ; W^{1, \infty}(\Omega)\right)} \leq C h\|\eta\|_{L^{2}\left(0, T ; W^{1, \infty}(\Omega)\right)} \\
& \leq \quad(3.41)
\end{aligned}
$$


In view of (3.8), (2.19), (1.2a) and (2.17) we deduce that

$$
\begin{array}{r}
\left|\int_{0}^{T}\left(\sigma_{\delta}^{h}\left(U_{\delta}^{-}\right) \nabla \Phi_{\delta}^{+}, \nabla\left(I-\pi^{h}\right) \eta\right) \mathrm{d} t\right|+\left|\int_{0}^{T}\left(\alpha^{h}\left(U_{\delta}^{-}\right) \nabla U_{\delta}^{+}, \nabla\left(I-\pi^{h}\right) \eta\right) \mathrm{d} t\right| \\
+\left|\int_{0}^{T} \int_{\Gamma_{N}^{u}}\left(\pi^{h} \gamma\right)\left(U_{\delta}^{+}-\pi^{h} \bar{u}_{N}\right)\left(I-\pi^{h}\right) \eta \mathrm{d} s \mathrm{~d} t\right| \\
+\left|\int_{0}^{T}\left(\left[\sigma_{\delta}^{h}\left(U_{\delta}^{-}\right)\right]^{\frac{1}{2}} \nabla \Phi_{\delta}^{+}\left[\left[\left(I-\pi^{h}\right) \eta\right] \mathcal{I}+\left(\left(\pi^{h} \eta\right) \mathcal{I}-D\left(\pi^{h} \eta\right)\right)\right]\left[\sigma_{\delta}^{h}\left(U_{\delta}^{-}\right)\right]^{\frac{1}{2}} \nabla \Phi_{\delta}^{+}\right) \mathrm{d} t\right| \\
\leq C\left[\left\|\left(I-\pi^{h}\right) \eta\right\|_{L^{\infty}\left(0, T ; W^{1, \infty}(\Omega)\right)}+h\left\|\pi^{h} \eta\right\|_{L^{\infty}\left(0, T ; W^{1, \infty}(\Omega)\right)}\right] .
\end{array}
$$

Combining (3.4b), (3.40), (3.41), (3.42), (2.19), (3.29), (3.27), (3.7c,d), (3.6d,e), (1.2a) and the denseness of $H^{1}\left(0, T ; W^{1, \infty}(\Omega)\right)$ in $L^{2}\left(0, T ; H^{1}(\Omega)\right) \cap H^{1}\left(0, T ; L^{2}(\Omega)\right) \cap L^{\infty}\left(\Omega_{T}\right)$ yields the desired result (3.36b).

We note that if one could establish uniqueness of the weak solution $\{\phi, u, v\}$ in Theorem 3.5, then convergence of the full sequence $\left\{\Phi_{\delta}^{+}, U_{\delta}, V_{\delta}\right\}_{h}$ would follow immediately. However, the lack of a uniqueness proof restricts us to subsequence convergence.

\section{NuMERICAL RESULTS}

Before presenting some numerical results in two space dimensions, we briefly state algorithms for solving the resulting system of algebraic equations for $\left\{\Phi_{\delta}^{n}, U_{\delta}^{n}, V_{\delta}^{n}\right\}$ arising at each time level from the approximation $\left(\mathrm{P}_{\delta}^{h, \tau}\right)$. As (2.18a) is independent of $\left\{U_{\delta}^{n}, V_{\delta}^{n}\right\}$, we first solve the resulting linear equation to obtain $\Phi_{\delta}^{n}$. To this end we employ a preconditioned conjugate gradient method.

Adopting the obvious notation, the system (2.18b) can be rewritten as: find $\left\{\underline{U}_{\delta}^{n}, \underline{V}_{\delta}^{n}\right\} \in \mathbb{R}^{\mathcal{J}} \times \mathbb{R}^{\mathcal{J}}$ such that

$$
M \underline{V}_{\delta}^{n}+\tau_{n} A^{n-1} \underline{U}_{\delta}^{n}=\underline{r} \quad \text { and } \quad \underline{V}_{\delta}^{n} \in \rho\left(\underline{U}_{\delta}^{n}\right) ;
$$

where $M$ and $A^{n-1}$ are symmetric $\mathcal{J} \times \mathcal{J}$ matrices, $\mathcal{J}:=\# J$, with entries

$$
\begin{aligned}
& M_{i j}:=\left(\chi_{i}, \chi_{j}\right)^{h}, \quad A_{i j}^{n-1}:=\left(\alpha^{h}\left(U_{\delta}^{n-1}\right) \nabla \chi_{i}, \nabla \chi_{j}\right)+\int_{\Gamma_{N}^{u}} \pi^{h}\left[\gamma \chi_{i} \chi_{j}\right] \mathrm{d} s \\
& \text { and } \quad \underline{r}:=M \underline{V}_{\delta}^{n-1}+\underline{s} \in \mathbb{R}^{\mathcal{J}}, \quad \underline{s}_{j}:=\left(\sigma_{\delta}^{h}\left(U_{\delta}^{n-1}\right) \nabla \Phi_{\delta}^{n}, D\left(\chi_{j}\right) \nabla \Phi_{\delta}^{n}\right)+\int_{\Gamma_{N}^{u}} \pi^{h}\left[\gamma \bar{u}_{N} \chi_{j}\right] \mathrm{d} s .
\end{aligned}
$$

A modified version of the standard SOR algorithm to solve (4.1), with a global convergence proof, can be found in [3]. We briefly describe the method here. Given $\underline{U}_{\delta}^{n, 0}$, for each $j=1 \rightarrow \mathcal{J}$ one has to solve

$$
M_{j j}\left[\underline{V}_{\delta}^{n, k}\right]_{j}+\tau_{n} A_{j j}^{n-1}\left[\underline{U}_{\delta}^{n, k}\right]_{j}=\underline{\widehat{r}}_{j}, \quad\left[\underline{V}_{\delta}^{n, k}\right]_{j} \in \rho\left(\left[\underline{U}_{\delta}^{n, k}\right]_{j}\right) ;
$$

where $\underline{\widehat{x}}_{j}$ depends on $\underline{r}, \underline{U}_{\delta}^{n, k-1}$ and already computed entries of $\underline{U}_{\delta}^{n, k}$. On obtaining the unique $\left[\underline{U}_{\delta}^{n, k}\right]_{j}$ from the simple nonlinear scalar equation (4.2a), we perform a relaxation step

$$
\left[\underline{U}_{\delta}^{n, k}\right]_{j}=\omega_{j}^{k}\left[\underline{U}_{\delta}^{n, k}\right]_{j}+\left(1-\omega_{j}^{k}\right)\left[\underline{U}_{\delta}^{n, k-1}\right]_{j}
$$

where $\omega_{j}^{k}=1$ if $\left[\underline{U}_{\delta}^{n, k}\right]_{j} \cdot\left[\underline{U}_{\delta}^{n, k-1}\right]_{j} \leq 0$ and $\omega_{j}^{k}=\omega \in(0,2)$ otherwise, and set

$$
\left[\underline{V}_{\delta}^{n, k}\right]_{j}=\frac{\widehat{\underline{\underline{r}}}_{j}-\tau_{n} A_{j j}^{n-1}\left[\underline{U}_{\delta}^{n, k}\right]_{j}}{M_{j j}} .
$$




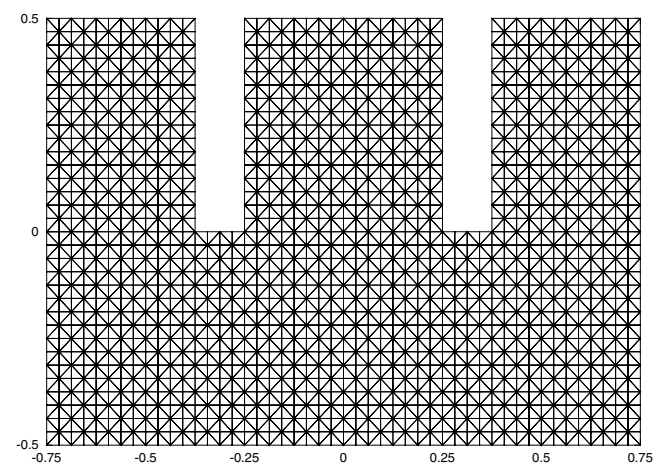

FiguRE 1. Triangulation of $\Omega$.

For the iterative algorithm $(4.2 \mathrm{a}-\mathrm{c})$ we set for $n \geq 1,\left\{U_{\delta}^{n, 0}, V_{\delta}^{n, 0}\right\} \equiv\left\{U_{\delta}^{n-1}, V_{\delta}^{n-1}\right\}$ and adopted the stopping criterion

with tol $=10^{-8}$

$$
\left|V_{\delta}^{n, k}-V_{\delta}^{n, k-1}\right|_{0, \infty}<t o l
$$

We chose $\Omega$ to be the domain $\left(-\frac{3}{4}, \frac{3}{4}\right) \times\left(-\frac{1}{2}, \frac{1}{2}\right) \backslash\left\{\left[-\frac{3}{8},-\frac{1}{4}\right] \times\left[0, \frac{1}{2}\right] \cup\left[\frac{3}{8}, \frac{1}{4}\right] \times\left[0, \frac{1}{2}\right]\right\}$ and for simplicity partitioned the domain into uniform right-angled isoceles triangles. An example triangulation of $\Omega$ can be seen in Figure 1. Obviously, a more accurate approximation can be obtained by using a finer mesh in the vicinity of the non-convex angles of $\Omega$ in order to approximate better the generated local gradient singularities in $\phi$ and $u$. The boundary data was chosen to be

$$
\bar{u}_{D} \equiv-1, \quad \bar{u}_{N} \equiv 1, \quad \gamma \equiv 1, \quad \text { and } \quad \bar{\phi}= \pm 1 \text { on } \Gamma_{ \pm}
$$

The initial data $v^{0}$ to $(\mathrm{P})$ was chosen such that $u^{0}=\psi\left(v^{0}\right)$ had the form

$$
u^{0}(x):=\min \{-1+\beta(x), 1\}, \quad \text { where } \quad \beta \in H_{u, 0}^{1}(\Omega) \cap W^{1, \infty}(\Omega) \text { with } \beta(x) \geq 0
$$

and $u^{0}=0$ on a curve. We then set $U_{\delta}^{0}=\pi^{h} u^{0}$ and $V_{\delta}^{0}\left(p_{j}\right) \in \rho\left(U_{\delta}^{0}\left(p_{j}\right)\right)$ for all $j \in J$ with $V_{\delta}^{0}\left(p_{j}\right)=\frac{1}{2} \lambda$ if $U_{\delta}^{0}\left(p_{j}\right)=0$. (4.3) models an initial temperature distribution between -1 and 1 ; satisfying the Dirichlet boundary conditions on $u$. In particular, we implemented, on recalling that $x=\left(x_{1}, x_{2}\right)$ with $x_{1}$ the horizontal variable,

$$
\text { (i) } \beta(x):=\left\{\begin{array}{ll}
20 \max \left\{x_{2}-\frac{2}{5}, 0\right\} & \text { if }\left|x_{1}\right| \leq \frac{1}{4}, \\
0 & \text { otherwise; }
\end{array} \quad \text { and } \quad(\text { ii }) \quad \beta(x):=20 \max \left\{\frac{1}{4}-r(x), 0\right\}\right.
$$

where $r(x):=\left(\frac{1}{4} x_{1}^{2}+x_{2}^{2}\right)^{\frac{1}{2}}$. (4.3) $(i)$ gives rise to a strip between the two electrodes as the initial conducting region, $\left\{u^{0}>0\right\}$, see the first plot in Figure 2; whilst (4.3)(ii) gives rise to an elliptical region, see the first plot in Figure 5. For the other data (1.3), (1.5) and (1.7) to (P) we chose $\rho_{ \pm}=\lambda=1, \alpha \equiv 1, s_{0}=1, p=2$. In all the experiments below we plot the contour $V_{\delta}(, x, t)=0$ at different times $t$ in order to see the evolution of the conducting region.

Our first experiment shows the evolution of a conducting strip between the electrodes, i.e. $u^{0}$ is given by (4.3) $(i)$, without $\left(\sigma_{0}=0\right)$ and with $\left(\sigma_{0}=5\right)$ the effect of Joule heating, respectively, until $T=5$.

For the experiment with $\sigma_{0}=5$ we investigated convergence of our approximation by starting with $h=\frac{\sqrt{2}}{32}$, $\tau=\delta=2 \times 10^{-2}$, see Figure 2, and successively halving the parameters $h, \tau$ and $\delta$ and checking agreement between the contours on successive meshes. We are satisfied that the results obtained for the choice $h=\frac{\sqrt{2}}{128}$, 

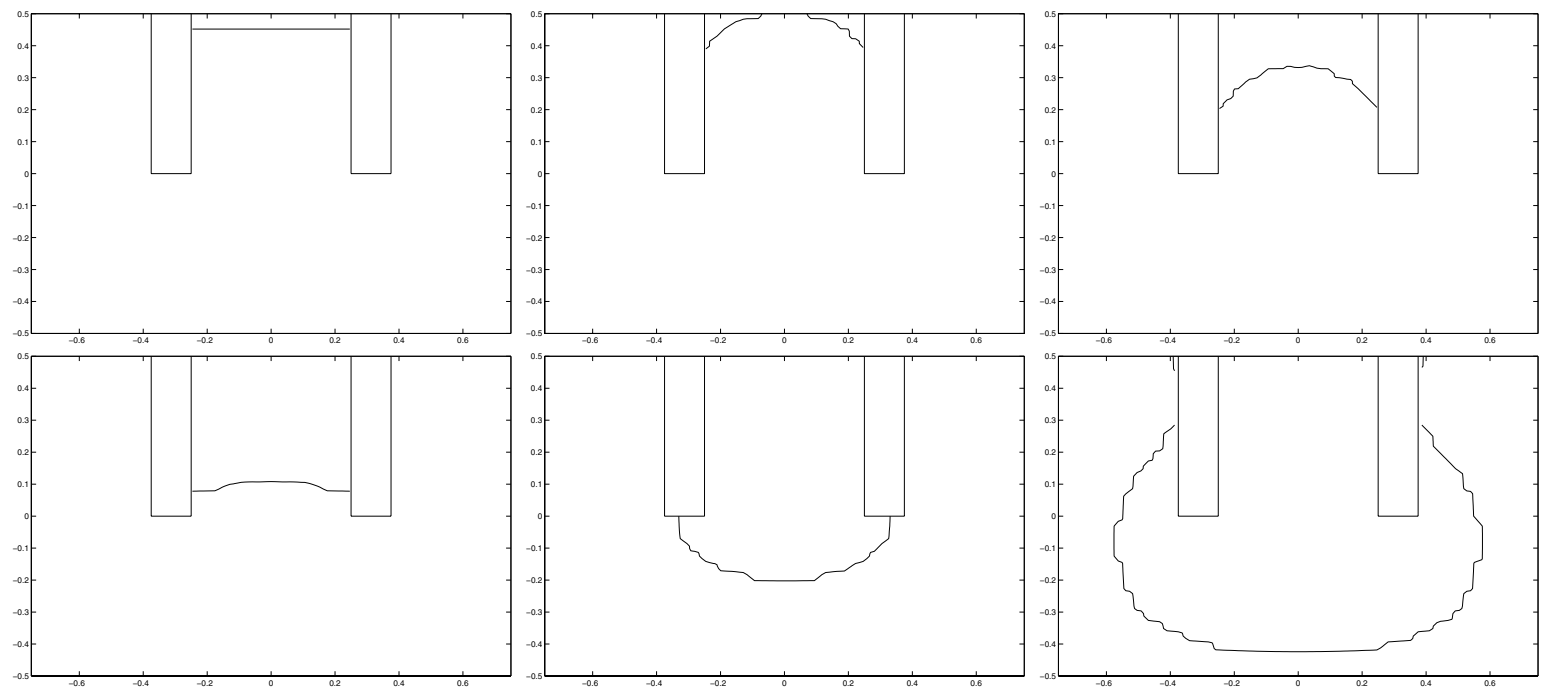

Figure 2. $\left(\sigma_{0}=5\right)$ Contour plot for $V_{\delta}(x, t)=\frac{\lambda}{2}$ at times $t=0,0.1,0.3,0.5,0.6,5$.
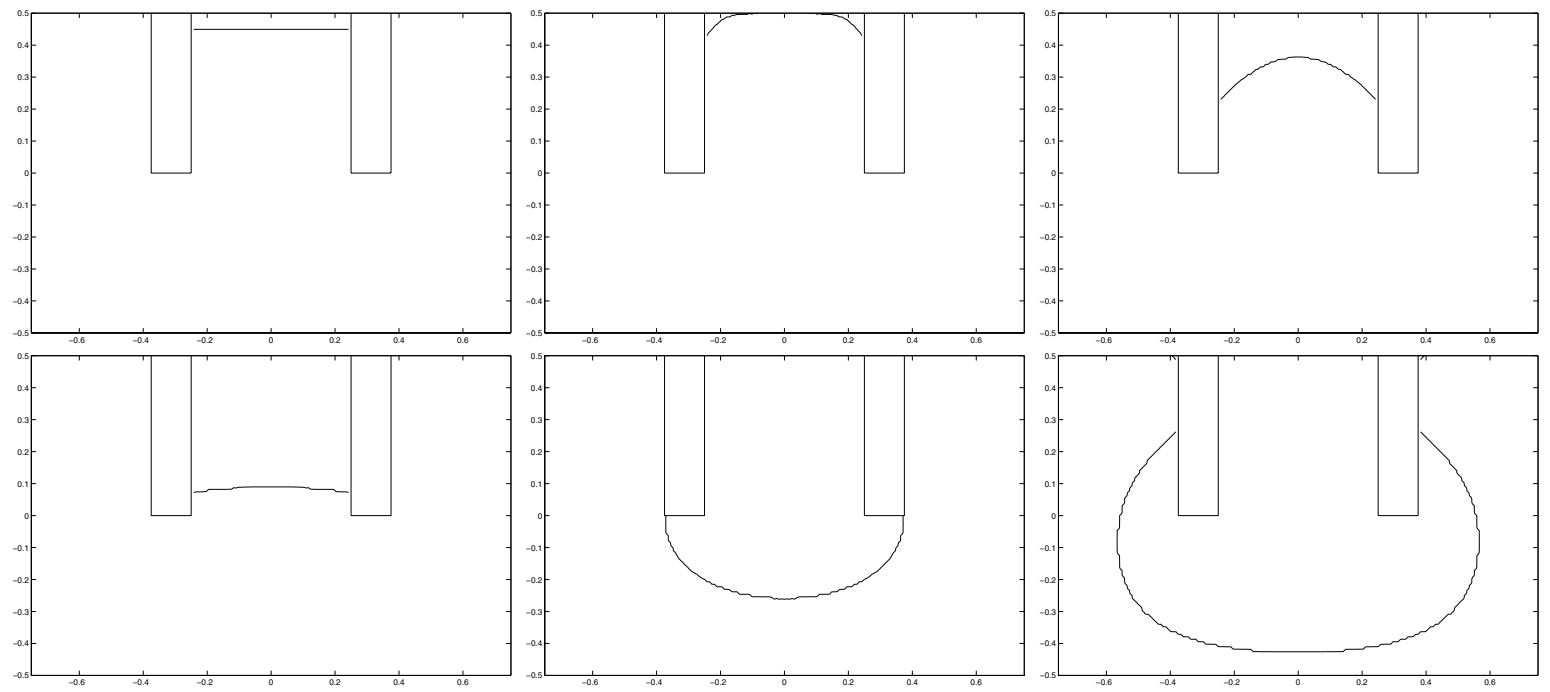

Figure 3. $\left(\sigma_{0}=5\right)$ Contour plot for $V_{\delta}(x, t)=\frac{\lambda}{2}$ at times $t=0,0.1,0.3,0.5,0.6,5$.

$\tau=\delta=5 \times 10^{-3}$ show a converged solution, see Figure 3. For the above crude and fine choices of $h$ we chose $\omega=1.7$ and 1.85, respectively, for the iterative algorithm $(4.2 \mathrm{a}-\mathrm{c})$. The plot for $t=T$ is very close to a steady state. In Figure 4 we repeat the experiment with the fine mesh parameters in the case of no Joule heating $\left(\sigma_{0}=0\right)$ being present, with all remaining parameters fixed as before. Again, the plot for $t=T$ is very close to a steady state. Comparing Figures 3 and 4, we see the effect of Joule heating on the conducting/molten region.

Our second experiment, see Figure 5, is with all parameters, including the mesh parameters, the same as for Figure 3; that is, with Joule heating being present $\left(\sigma_{0}=5\right)$, but now with the initial data (ii) in (4.3) as opposed to $(i)$. We note that the quasi steady states in Figures 3 and 5 are very similar, despite very different initial data. Furthermore, we note that the approximation with $D(\chi)$ in $(3.4 \mathrm{~b})$ replaced by $\chi$ yielded graphically indistinguishable results throughout. 

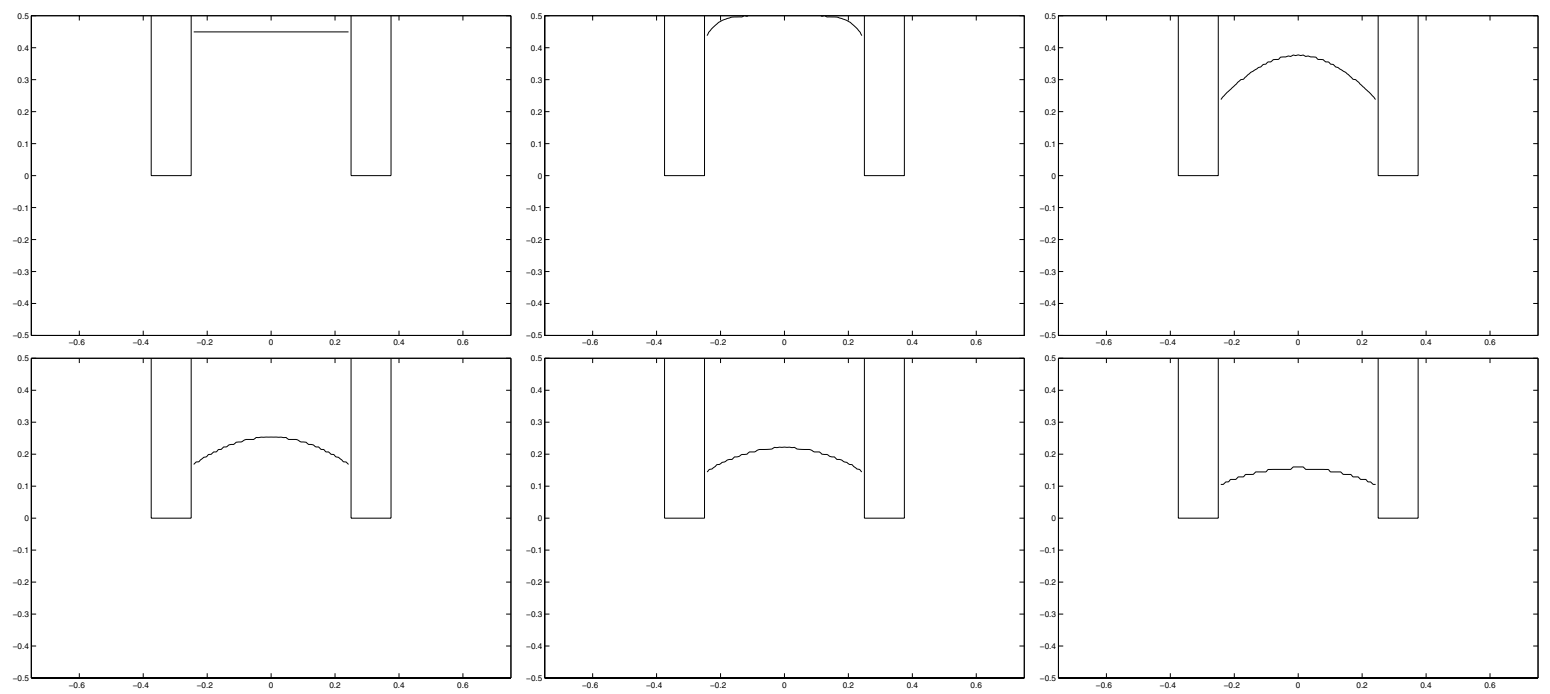

Figure 4. $\left(\sigma_{0}=0\right)$ Contour plot for $V_{\delta}(x, t)=\frac{\lambda}{2}$ at times $t=0,0.1,0.3,0.5,0.6,5$.
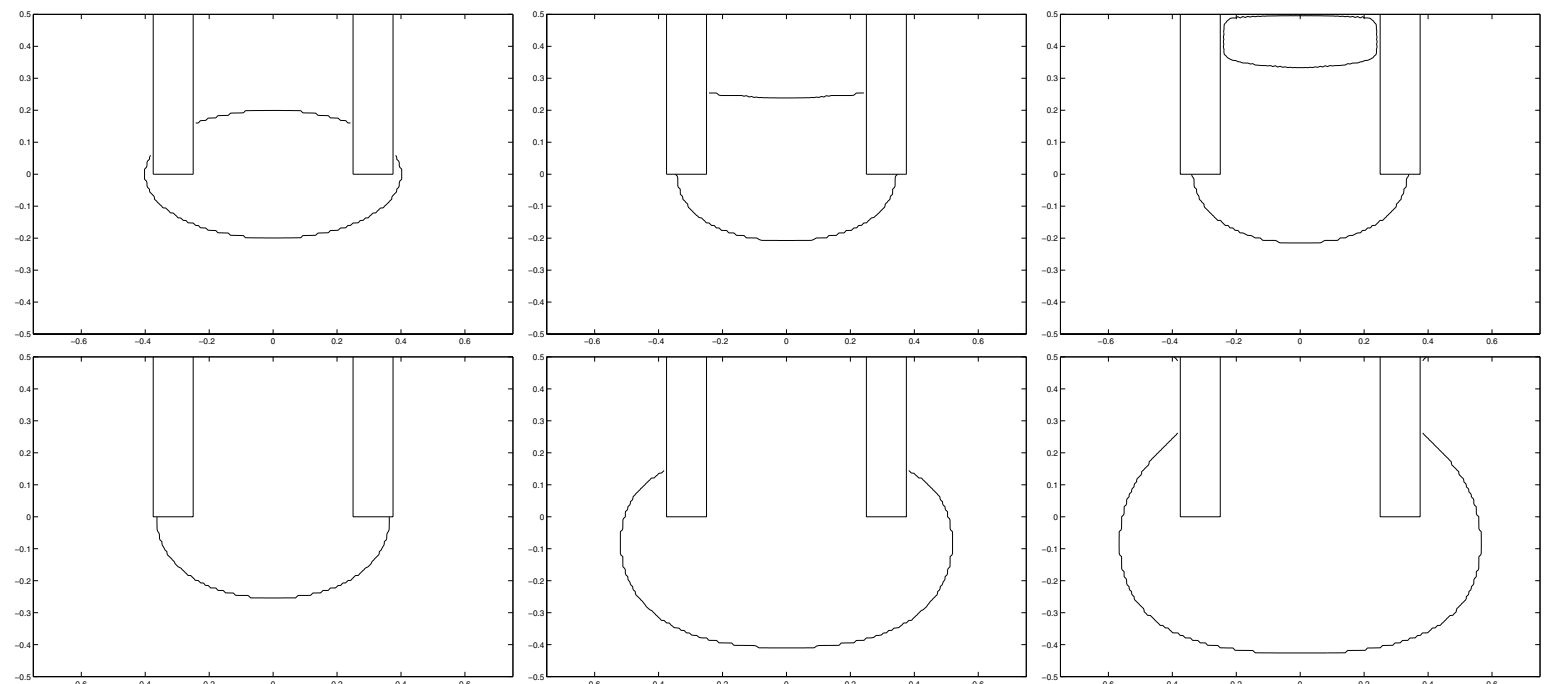

Figure 5. $\left(\sigma_{0}=5\right)$ Contour plot for $V_{\delta}(x, t)=\frac{\lambda}{2}$ at times $t=0,0.025,0.05,0.1,0.3,5$.

\section{REFERENCES}

[1] J.W. Barrett and C.M. Elliott, A finite element method on a fixed mesh for the Stefan problem with convection in a saturated porous medium, in Numerical Methods for Fluid Dynamics, K.W. Morton and M.J. Baines Eds., Academic Press (London) (1982) 389-409.

[2] J.W. Barrett and R. Nürnberg, Convergence of a finite element approximation of surfactant spreading on a thin film in the presence of van der Waals forces. IMA J. Numer. Anal. 24 (2004) 323-363.

[3] C.M. Elliott, On the finite element approximation of an elliptic variational inequality arising from an implicit time discretization of the Stefan problem. IMA J. Numer. Anal. 1 (1981) 115-125.

[4] C.M. Elliott, Error analysis of the enthalpy method for the Stefan problem. IMA J. Numer. Anal. 7 (1987) 61-71. 
[5] C.M. Elliott and S. Larsson, A finite element model for the time-dependent Joule heating problem. Math. Comp. 64 (1995) 1433-1453.

[6] R.F. Gariepy, M. Shillor and X. Xu, Existence of generalized weak solutions to a model for in situ vitrification. European J. Appl. Math. 9 (1998) 543-559.

[7] S.S. Koegler and C.H. Kindle, Modeling of the in situ vitrification process. Amer. Ceram. Soc. Bull. 70 (1991) $832-835$.

[8] J. Simon, Compact sets in the space $L^{p}(0, T ; B)$. Ann. Math. Pura. Appl. 146 (1987) 65-96.

[9] X. Xu, A compactness theorem and its application to a system of partial differential equations. Differential Integral Equations 9 (1996) 119-136.

[10] X. Xu, Existence for a model arising from the in situ vitrification process. J. Math. Anal. Appl. 271 (2002) 333-342.

To access this journal online: www.edpsciences.org 